\title{
Geochemistry of Sedimentary Rocks from the Nkapa Formation, North Western Part of the Douala Basin, Cameroon: Implications for Provenance, Tectonic Setting and Paleoenvironmental Conditions
}

\author{
Florence Njinto Kwankam ${ }^{1}$, Christopher Mbaringong Agyingi², Josepha Foba-Tendo ${ }^{3}$, \\ Cheo Emmanuel Suh ${ }^{2,4}$
}

${ }^{1}$ Department of Petroleum Engineering, National Higher Polytechnic Institute (NAHPI), The University of Bamenda, Bambili, North West Region, Cameroon

${ }^{2}$ Department of Geology, Faculty of Science, The University of Bamenda, Bambili, North West Region, Cameroon

${ }^{3}$ Department of Chemistry, University of Buea, Buea, South West Region, Cameroon

${ }^{4}$ Department of Geology, University of Buea, Buea, South West Region, Cameroon

Email: ^florencenjito@yahoo.com

How to cite this paper: Kwankam, F.N., Agyingi, C.M., Foba-Tendo, J. and Suh, C.E. (2021) Geochemistry of Sedimentary Rocks from the Nkapa Formation, North Western Part of the Douala Basin, Cameroon: Implications for Provenance, Tectonic Setting and Paleoenvironmental Conditions. International Journal of Geosciences, 12, 739-762.

https://doi.org/10.4236/ijg.2021.129042

Received: July 7, 2021

Accepted: August 29, 2021

Published: September 1, 2021

Copyright ( 2021 by author(s) and Scientific Research Publishing Inc. This work is licensed under the Creative Commons Attribution International License (CC BY 4.0).

http://creativecommons.org/licenses/by/4.0/ (c) (i) Open Access

\begin{abstract}
The Douala sedimentary basin in Cameroon is the main gas prospective basin in the country and has witnessed a number of investigations in this regard. The Nkapa Formation is one of the basin's principal rock units and here we analyzed samples from sediments comprising this formation in order to constrain their provenance, tectonic setting and depositional environment using geochemical traits. Major and trace elements were analyzed by inductively coupled plasma mass spectrometry. Elemental ratios provide information on geochemical signatures of $\mathrm{Eu} / \mathrm{Eu}$ ratios of the sandstones and shales, suggesting the provenance of the sediments was felsic from continental rock protoliths. Calculated $\mathrm{K}_{2} \mathrm{O} / \mathrm{Na}_{2} \mathrm{O}$ ratios $>1$ is reminiscent of quartz-rich sediments deposited in passive margin (PM) environments with significant terrigenous input. The elemental ratios $\mathrm{La} / \mathrm{Sc}, \mathrm{La} / \mathrm{Ca}, \mathrm{Th} / \mathrm{Sc}$, and $\mathrm{Th} / \mathrm{Co}$ suggest that the sediment sources are felsic and mafic in nature. The clastic sediments were normalized using North American Shale Composite (NASC). The Ni/Co and $\mathrm{V} /(\mathrm{V}+\mathrm{Ni})$ ratios reveal that the sediments were deposited under variable conditions including euxinic, dysoxic and anoxic. Overall, the evidence from discrimination diagrams using major and trace elements suggest that the sediments were derived from mostly Passive margins (PM) and continental island arc (CIA) with minor Oceanic island arch (OIA) sources. Limestones from the studied area at Kompina, rich in calcium carbonate above $98.5 \%$
\end{abstract}


make them suitable for use in the chemical industries. In addition the felsic and mafic source rocks can lead to formation of light and dark colored minerals which can be of economic value.

\section{Keywords}

Geochemistry, Sedimentary Rocks, Nkapa Formation, Provenance, Depositional Environment

\section{Introduction}

Sedimentary rocks provide vital information on the provenance of sediments and for reconstructing the history of basins [1]. Geochemical signatures of sedimentary rocks furnish vital information for distinguishing tectonic settings, climates and depositional environments associated with sedimentary basins [2] [3] [4] [5] [6]. Significant chemical changes commonly occur during the transportation of sediments. However, although hydraulic sorting, weathering and diagenesis alter the geochemical composition of sediments, original source terrain signatures are often retained [5] [7]. The effects of these processes also largely depend on the duration between erosion and deposition [5]. Even though major and trace element geochemistry has been exploited mainly for sediment provenance and tectonic setting studies, when integrated with compositional data of rocks, such as carbonates, their application can be extended to depositional environments [8] [9].

Sediment provenance can be evaluated using geochemical approaches, especially discrimination diagrams. These diagrams, which are based on relationships between elements have significantly enhanced knowledge on sediment provenance and tectonic settings [7] [10] [11] [12] [13]. The sedimentology and petrochemistry of sedimentary strata of Tertiary age in West Africa have attracted worldwide attention in recent years for various reasons. These include the petroleum potential of these strata that lie in the eastern margin of the Atlantic Ocean [14] and the fact that carbonate resources for industrial use are common in these strata.

Several studies have been carried out in the Douala Basin over the years [14]-[23]. These previous studies have focused on the petroleum prospectivity of the sediments. The petrochemistry, provenance and environment of deposition of sedimentary rocks have not witnessed the same level of scientific scrutiny. In the present study, the major and trace element signatures of sedimentary rocks in the Tertiary Nkapa Formation are used to investigate the sediment sources and tectonic setting of the depositional environment.

The study area (Kompina) is located between latitudes $4^{\circ} 20^{\prime}$ and $4^{\circ} 24^{\prime} \mathrm{N}$ and longitudes $9^{\circ} 35^{\prime}$ and $9^{\circ} 40^{\prime} \mathrm{E}$ (Figure 1). It is part of the northern Douala Basin, which lies on the north-eastern flank of Mount Cameroon, a major volcanic horst in West-Central Africa [24] [25]. 


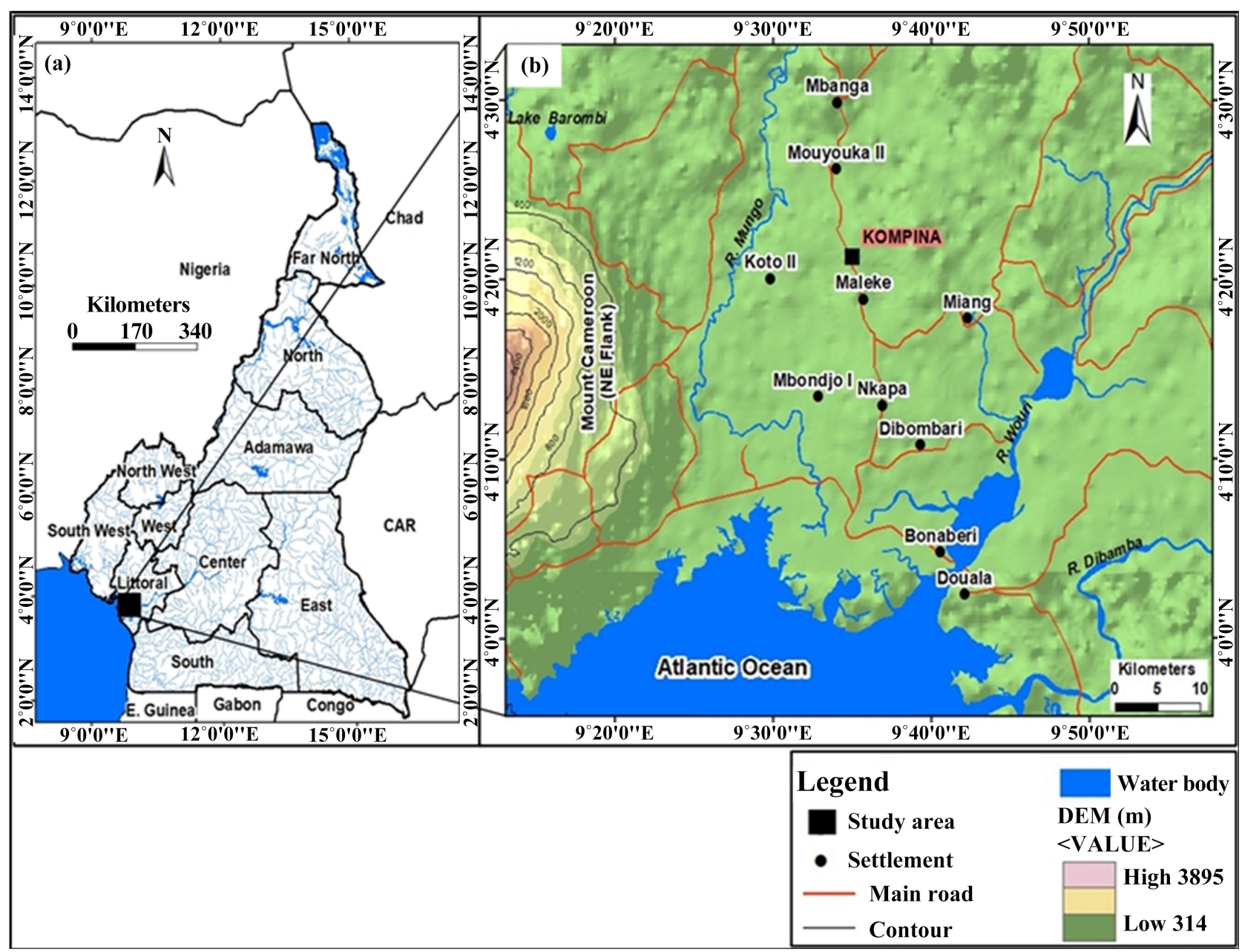

Figure 1. Location of the study area (Kompina) including (a) map of Cameroon (not to scale) highlighting the location of the study area within the Douala Basin in the Littoral Region and (b) map of the study area and its relief (pink represents hilly and mountainous areas, while yellow to green shows low-lying areas) northeast of Mount Cameroon, modified from [26].

\section{Origin, Evolution and Stratigraphy of the Douala Basin}

The Douala Basin (Figure 2) in Cameroon is a coastal sedimentary basin situated at the edge of the Gulf of Guinea [27]. Other coastal sedimentary basins in Cameroon include the Campo and the Rio del Rey Basins, and these three existed as a continuous basin during the Cretaceous period. The Tertiary volcanism that formed the Mount Cameroon volcanic axis (Cameroon volcanic line; CVL) separated the Rio del Rey from the Douala Basin [15] [28].

The origin of the Douala Basin is intimately linked to the rifting that created the South Atlantic Ocean during the Mesozoic ( 130 Ma) breakup of Gondwanaland [26]. The opening of the South Atlantic Ocean involved counter-clockwise rotation of the southern part of the African plate relative to South America around a pole centered in the North Atlantic. This produced shearing between the South and North African sub-plates in the Benue Trough and Central African Rift System or Central African Shear Zone (CASZ). 


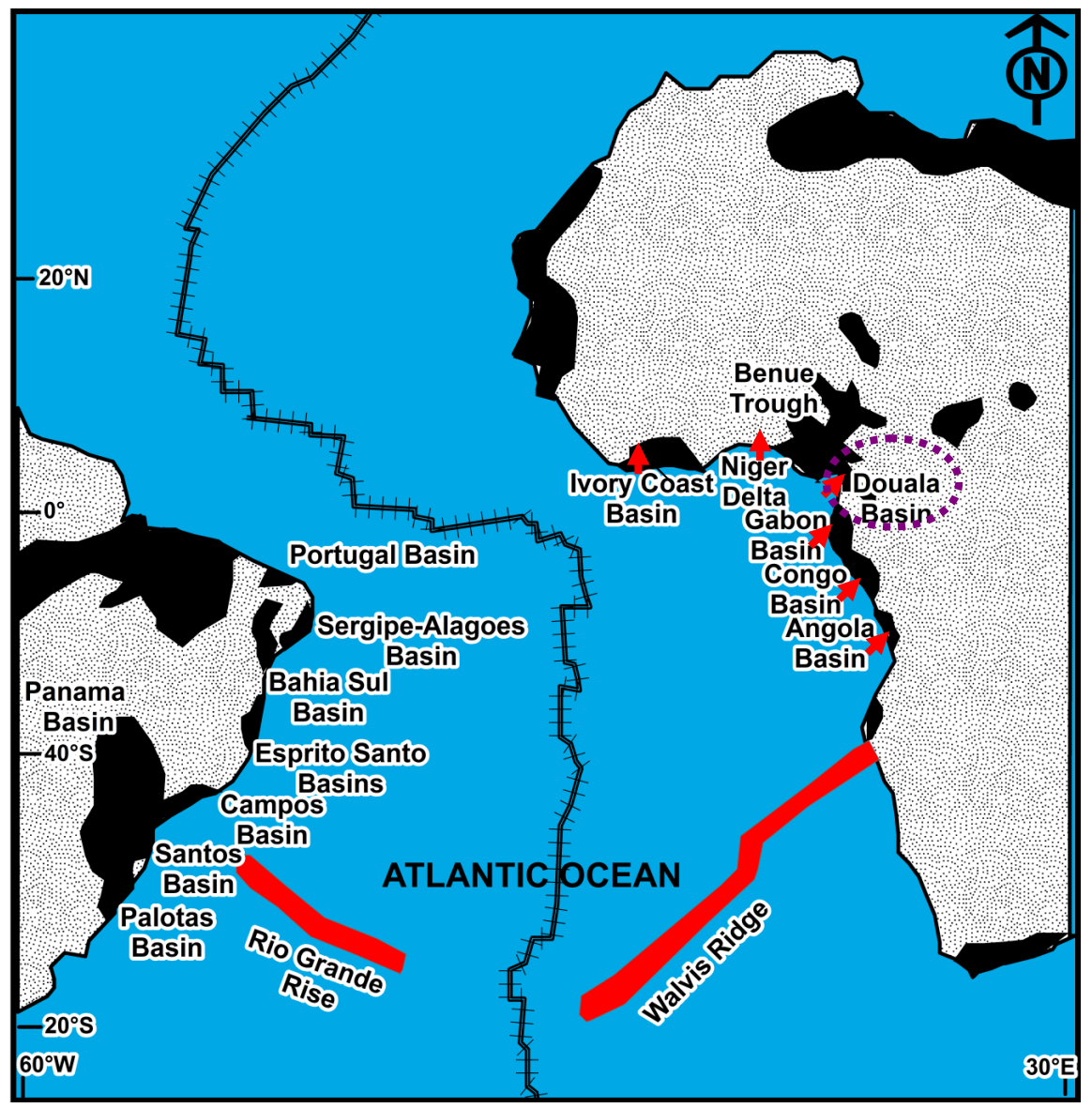

Figure 2. Map showing Sub-basins associated with rift systems in the South Atlantic Ocean i.e., basins of West Africa and Eastern Brazil (Brazil: Bahia Sul and Sergipe-Alagoas; West Africa: Congo, Gabon and Douala Basins (after [29]).

According to [29], by the Mid Aptian, the Cameroon margin experienced a major structural reconfiguration, which started with oceanic transform faulting and margin extensions, and this segmented the rift structure of the margin. The north boundary of the basin is the south edge of the Niger Delta province [30], while in the south, the boundary is the Early Cretaceous to Holocene Walvis Ridge volcanic high [17]. These basins are classified as Atlantic-type sag basins [31] and they contain Palaeozoic to Holocene strata. The opening of the South Atlantic is linked with the formation of salt basins, and the associated tectonic activities occurred during pre-rift, syn-rift and post-rift stages. These tectonic movements affected basins from the south to the north Atlantic margin including West Africa.

The Douala basin belongs to the southern section of the Cameroon shelf, which comprises extensive Cenozoic and Mesozoic deposits, while the northern section of the shelf is dominated by Late Pleistocene and Holocene units [32]. The Precambrian basement is overlain by Albian-Senonian, Palaeocene, Mid-Late Miocene, and Pliocene-Pleistocene units [33]. The deposition and preservation of the Cenozoic and Mesozoic sediments were controlled by faulting, which created depressions and related structures in the Precambrian basement [34]. De- 
formation types characterizing the structures of this margin include the following:

1) N-S-trending fractures parallel to the Precambrian onshore that formed during the Cretaceous rifting period, and these were crosscut by $\mathrm{N} 60^{\circ} \mathrm{E}$-trending cross-faults induced by the transform movement associated with the creation of the South Atlantic.

2) Structures that were governed by local comprehensive stresses during the Eocene transcurrent re-activation along the $\mathrm{N} 60^{\circ} \mathrm{E}$ transform fault, which extended the salt-controlled deformation.

3) Nearly N-S faults, which affected the Miocene-Pliocene or Quaternary nonfolded sequences, and which highlight distinct conditions [29].

Tectonic forces control the structural patterns in a basin [34], and thus, influence its sedimentary fill (volume and thickness of sediments). During the rifting in the South Atlantic, both the African and South American margins experienced extensional tectonics. These involved ocean ward movements of the inland Hinge, the Atlantic Hinge and the continental oceanic boundary (COB). The impacts of extensional tectonics are evident in the Douala Basin, although foreshortened by transcurrent (strike-slip) faulting [15]. The Atlantic Hinge was masked by high volumes of sediments delivered to the coast by rivers, and a modern shoreline has propagated across the previous deep-water setting. Growth structures (younging structures) akin to those in the onshore Niger Delta represent targets e.g. reservoirs in the Douala Basin of Cameroon. These structures were produced by shale diapirism in the delta and displacement by squeezing of sediments piling ocean ward [35]. The frontal toe thrust sediments of the delta complex are associated with upfolds [36] [37]. The COB extends between 150 - $250 \mathrm{~km}$ in water characterized by depths exceeding $2.5 \mathrm{~km}$, and it is closest to the Atlantic Hinge in the transcurrent faults regions.

Environments of deposition in the Douala Basin vary from continental at the base to evaporitic and marginal at the top and these reflect the progression from a rift valley to a passive margin [38]. The stratigraphy of the Douala Basin has been reported in detail in previous studies [16] [17] [18] [19] [29] [39] [40] [41] [42]. Formations in the basin are progressively younger from the north to the south of the basin. Figure 3 is a generalized stratigraphic section adapted from [16] showing the formations and rock types in the basin as well as their ages. These formations overlie a highly faulted Precambrian basement, which represents the oldest rocks in the basin (Figure 3). In vertical succession, formations in the basin include the following: Wouri, Matanda, Souellaba, Nkapa, Logbaba, Logbadjeck, Mungo and Mundek, which vary in age from Tertiary to Cretaceous.

The Mungo Formation dominantly contains sandstones, with subordinate intercalated limestones and shales as well as minor marls, silty clays and siltstones. The thick unfossiliferous sandstones of this formation, which have been assigned to the Lower Turonian, overlie the Mundeck Formation which rest on the crystalline basement. The Logbajeck and the Logbaba Formations are considered as one formation (Logbaba Formation) in some studies and attributed to the Upper 


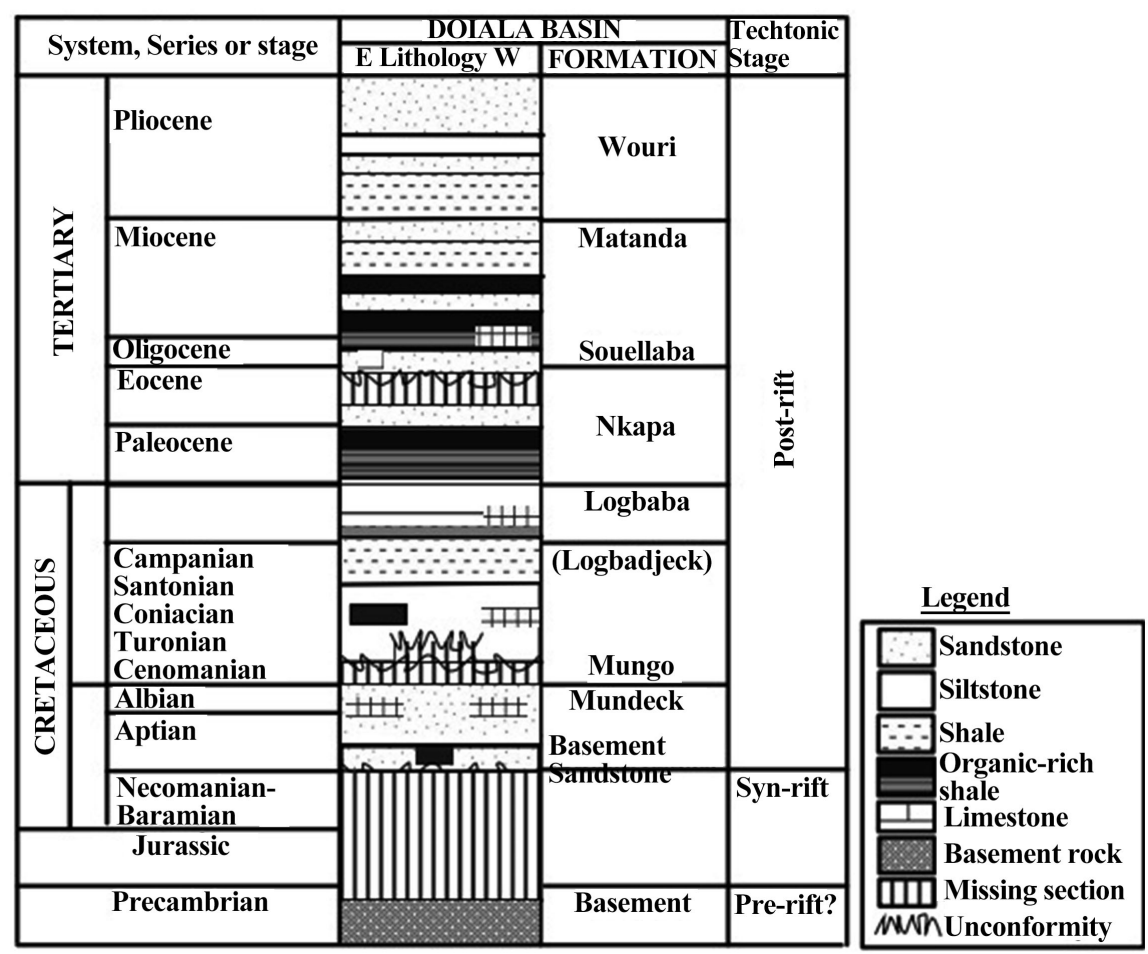

Figure 3. Generalised stratigraphic columns showing age, lithology, formation name and tectonic stage for sediments in the Douala Basin (modified after [16]).

Cretaceous [17]. This formation comprises shales, sandstones and localized alternations of carbonates, sandstones and shales. The Tertiary Nkapa Formation, which represents the study area, is characterized as a shale-dominated formation [17]. However, it also comprises sandstones, limestones, siltstones, marl and mudstones. In the offshore part of the basin, the Nkapa Formation represents a mud-dominated channel levee system. In this section, the formation also comprises of organic rich shales, limestones, siltstones and calcareous mudstones [16].

The Souellaba and Matanda Formations are also of Tertiary age. The Oligocene Souellaba Formation is made up of sandstones, volcanic fragments, claystones, and carbonates. The presence of volcanic components is attributed to activities of the CVL. The Pleistocene Wouri Formation corresponds to the presently developing deltaic section. The stratigraphic pile involves sands, claystones and shales. The stratigraphic column of the Douala Basin contains mainly arenaceous, argillaceous and carbonate rocks.

\section{Description of Samples and Analytical Methods}

Field study consisted of investigating rock outcrops in the study area. A systematic mapping and sampling of lithologic units in the Nkapa Formation, outcropping mainly along the banks of the Bongue River and its tributaries, river Channels, road cuts and valleys at Kompina, Lingue, Nkolwongo and kesso localities using topographic map delineating the study area (scale of 1:50.000). Field study involved observations, detailed description and measurements of li- 
thologic sections in the studied area as well as taking GPS readings for sample location points and collecting representative samples for analysis.

A total of forty (40) representative samples were selected for geochemical analysis from the study area. The comprised dominantly carbonate rocks (seventeen (17) Limestones and three (3) marly limestones; five (5) calcareous shales; five (5) sandstones and one (1) lateritic soil sample (Figure 1 and Figure 2).

Major and trace element compositions of the samples were determined in ACME Laboratory in Vancouver, Canada, using inductively coupled plasma-mass spectrometry (ICP-MS). The procedure involved fusing about 0.25 grams of samples in a crucible for 30 minutes at high temperature $\left(750^{\circ} \mathrm{C}-800^{\circ} \mathrm{C}\right)$, using lithium metaborate. Insulated gloves and a crucible tongs are used to remove the crucible after heating and the heated sample allowed cooling at room temperature, followed by acid dissolution with $10 \mathrm{~mL}$ of concentrated hydrochloric acid that is mixed with $20 \mathrm{ml}$ of high purity water. The sample in the crucible is mixed with hydrochloric acid and allowed to thoroughly dissolve. The solution is mixed by inversion and allowed to sit undisturbed for at least 30 minutes. This allows graphite to settle to the bottom and the solution left is ready for measurement. The accuracy of the measurements for major oxides, trace elements and rare earth elements (REEs) range between $0.002-0.04,0.0-0.5$ and $0.01-1$, respectively.

To examine the geochemical characteristics of the carbonates, shales and sandstones in the Nkapa Formation, data generated were normalized using the North American Shale Composite (NASC) [5] for trace elements.

\section{Results}

The major and trace element data for carbonates (limestones and marly limestones), shales, calcareous shales and sandstones from the Nkapa Formation in the Douala Basin investigated are presented in Table 1 and Table 2, with just one lateritic soil sample being analysed.

Table 1. Major oxides (wt\%), trace and rare-earth elements concentrations (ppm) of limestones and marly limestones.

\begin{tabular}{|c|c|c|c|c|c|c|c|c|c|c|c|c|c|c|c|c|c|c|c|c|}
\hline \multirow{2}{*}{$\begin{array}{c}\text { Rock } \\
\text { sample } \\
\text { No. }\end{array}$} & \multicolumn{17}{|c|}{ Limestones } & \multicolumn{3}{|c|}{ Marly limestones } \\
\hline & S1 & S4 & S7 & S10 & S14 & S16 & S18 & $\mathrm{S} 20$ & S22 & S24 & S26 & S27 & S34 & S35 & S36 & S37 & $\mathrm{S} 40$ & S3 & S6 & S13 \\
\hline $\mathrm{SiO}_{2}$ & 21.10 & 2.43 & 35.5 & 10.56 & 12.3 & 17.2 & 24.6 & 5.18 & 22.5 & 13.9 & 26.3 & 17.7 & 19.0 & 23.2 & 34.0 & 31.3 & 4.32 & 45.5 & 37.46 & 54.31 \\
\hline $\mathrm{TiO}_{2}$ & 0.94 & 0.07 & 1.47 & 0.28 & 0.31 & 0.57 & 0.8 & 0.16 & 0.76 & 0.46 & 0.99 & 0.69 & 0.48 & 0.70 & 1.05 & 0.83 & 0.16 & 0.32 & 1.02 & 0.19 \\
\hline $\mathrm{Al}_{2} \mathrm{O}_{3}$ & 5.16 & 0.98 & 8.09 & 3.73 & 3.12 & 3.97 & 5.72 & 1.55 & .15 & 3.64 & 5.45 & 4.15 & 2.57 & 3.69 & 6.74 & 6.07 & 1.27 & 4.94 & 7.95 & 3.5 \\
\hline $\mathrm{Fe}_{2} \mathrm{O}_{3}$ & 6.28 & 2.17 & 10.68 & 7.38 & 5.96 & 4.9 & 5.77 & 4.16 & 6.80 & 5.19 & 7.34 & 6.96 & 5.24 & 6.10 & 5.95 & 6.27 & 3.76 & 3.77 & 6.70 & 3.09 \\
\hline $\mathrm{MnO}$ & 0.08 & 0.06 & 0.10 & 0.14 & 0.08 & 0.05 & 0.05 & 0.11 & 0.09 & 0.07 & 0.09 & 0.09 & 0.13 & 0.14 & 0.07 & 0.06 & 0.10 & 0.06 & 0.06 & 0.13 \\
\hline $\mathrm{MgO}$ & 1.05 & 0.75 & 1.88 & 1.82 & 1.26 & 1.34 & 1.33 & 1.34 & 1.39 & 7.79 & 1.37 & 1.30 & 6.33 & 8.19 & 1.30 & 1.23 & 1.01 & 0.67 & 1.24 & 0.6 \\
\hline $\mathrm{CaO}$ & 33.08 & 51.52 & 19.32 & 40.85 & 40.8 & 37.9 & 31.6 & 47.4 & 32.5 & 32.6 & 29.6 & 35.7 & 32.3 & 26.1 & 25.2 & 26.7 & 48.2 & 23.19 & 21.71 & 19.5 \\
\hline $\mathrm{Na}_{2} \mathrm{O}$ & 0.13 & 0.02 & 0.27 & 0.1 & 0.08 & 0.16 & 0.22 & 0.06 & 0.21 & 0.17 & 0.20 & 0.14 & 0.09 & 0.12 & 0.30 & 0.24 & 0.10 & 0.32 & 0.35 & 0.27 \\
\hline
\end{tabular}




\section{Continued}

\begin{tabular}{|c|c|c|c|c|c|c|c|c|c|c|c|c|c|c|c|c|c|c|c|c|}
\hline $\mathrm{K}_{2} \mathrm{O}$ & 1.39 & 0.09 & 1.88 & 0.37 & 0.39 & 0.96 & 1.44 & 0.16 & 1.27 & 0.80 & 1.48 & 0.90 & 0.39 & 0.58 & 1.57 & 1.48 & 0.22 & 1.04 & 2.38 & 0.84 \\
\hline $\mathrm{P}_{2} \mathrm{O}_{5}$ & 0.08 & 0.06 & 0.05 & 0.84 & 0.1 & 0.03 & 0.06 & 0.23 & 0.09 & 0.09 & 0.07 & 0.29 & 0.08 & 0.09 & 0.11 & 0.07 & 2.44 & 0.02 & 0.09 & $<0.01$ \\
\hline $\mathrm{Cr}_{2} \mathrm{O}_{3}$ & 0.01 & 0.01 & 0.02 & 0.016 & 0.01 & 0.01 & 0.01 & 0.01 & 0.01 & 0.01 & 0.01 & 0.01 & 0.01 & 0.01 & 0.01 & 0.01 & 0.00 & 0.004 & 0.01 & $<0.002$ \\
\hline LOI & 30.4 & 41.6 & 20.4 & 33.6 & 35.4 & 32.6 & 28.1 & 39.5 & 28.9 & 35.0 & 26.8 & 31.7 & 33.1 & 30.8 & 23.3 & 25.4 & 38.2 & 20 & 20.7 & 17.5 \\
\hline Total & 99.70 & 99.76 & 99.66 & 99.69 & 99.8 & 99.7 & 99.6 & 99.8 & 99.7 & 99.7 & 99.7 & 99.7 & 99.8 & 99.7 & 99.6 & 99.7 & 99.8 & 99.83 & 99.67 & 99.93 \\
\hline $\mathrm{Ni}$ & 25.00 & $<20$ & 40 & $<20$ & $<20$ & $<20$ & $<20$ & $<20$ & 23 & $<20$ & 25 & 21 & $<20$ & $<20$ & 23 & $<20$ & $<20$ & $<20$ & $<20$ & $<20$ \\
\hline Sc & 6.00 & 2 & 9 & 6 & 4 & 4 & 6 & 3 & 5 & 4 & 6 & 6 & 3 & 4 & 6 & 6 & 3 & 2 & 7 & 1 \\
\hline $\mathrm{Ba}$ & 454.00 & 148 & 548 & 158 & 110 & 341 & 436 & 56 & 386 & 228 & 488 & 264 & 116 & 190 & 526 & 452 & 69 & 321 & 719 & 275 \\
\hline $\mathrm{Be}$ & 1.00 & 1 & 2 & 2 & 2 & $<1$ & 1 & $<1$ & 2 & $<1$ & 1 & $<1$ & $<1$ & 1 & 6 & 2 & $<1$ & 3 & 4 & $<1$ \\
\hline Co & 13.90 & 2.2 & 24.1 & 19.7 & 7.6 & 13.3 & 17 & 4.1 & 16.0 & 9.1 & 18.8 & 14.7 & 5.1 & 6.6 & 14.9 & 11.1 & 3.6 & 5.5 & 17.3 & 2.6 \\
\hline Cs & 0.60 & 0.1 & 0.7 & 0.4 & 0.3 & 0.4 & 0.5 & 0.2 & 0.4 & 0.4 & 0.7 & 0.3 & 0.5 & 0.4 & 0.7 & 0.7 & $<0.1$ & 0.4 & 0.8 & 0.2 \\
\hline $\mathrm{Ga}$ & 6.40 & 0.7 & 12.7 & 7.7 & 5.5 & 6.4 & 8.6 & 3.1 & 7.7 & 5.4 & 9.2 & 6.8 & 3.7 & 5.8 & 10.4 & 8.7 & 1.6 & 4.9 & 10.8 & 3.9 \\
\hline $\mathrm{Hf}$ & 17.20 & 0.8 & 14.2 & 5.3 & 5 & 13 & 19.1 & 1.9 & 19.1 & 8.0 & 14.8 & 10.5 & 9.8 & 11.2 & 27.3 & 29.0 & 1.9 & 3.9 & 19.5 & 1.5 \\
\hline $\mathrm{Nb}$ & 18.00 & 3.3 & 33.5 & 12.3 & 11 & 17.1 & 22.6 & 6.2 & 21.8 & 15.9 & 25.4 & 18.1 & 12.6 & 17.8 & 25.0 & 26.7 & 7.6 & 8.1 & 30 & 5.7 \\
\hline $\mathrm{Rb}$ & 34.70 & 3.4 & 43.7 & 10.2 & 10.7 & 22.6 & 32.8 & 4.7 & 27.5 & 18.2 & 34.0 & 20.2 & 10.1 & 15.4 & 38.0 & 33.0 & 6.0 & 21 & 53.6 & 16.2 \\
\hline Sn & 2.00 & $<1$ & 3 & 2 & 1 & 3 & 2 & $<1$ & 2 & 1 & 2 & $<1$ & $<1$ & $<1$ & 2 & 2 & $<1$ & $<1$ & 3 & $<1$ \\
\hline $\mathrm{Sr}$ & 707.00 & 1143.3 & 592.2 & 1456 & 884 & 997 & 852 & 983 & 805 & 766 & 653 & 905 & 410 & 385 & 775 & 862 & 1000 & 581.3 & 567.8 & 417 \\
\hline $\mathrm{Ta}$ & 0.50 & 0.1 & 2.1 & 1.1 & 0.6 & 0.5 & 1.2 & 1.1 & 1.3 & 0.7 & 1.4 & 0.6 & 0.3 & 0.9 & 1.3 & 1.1 & $<0.1$ & 0.6 & 1.8 & 2 \\
\hline Th & 9.60 & 2.4 & 11.4 & 8.3 & 4.9 & 7.5 & 11.7 & 2.5 & 9.1 & 4.9 & 9.8 & 6.6 & 7.2 & 7.8 & 11.1 & 10.8 & 2.4 & 3.1 & 12 & 1.1 \\
\hline $\mathrm{U}$ & 2.50 & 0.4 & 2.1 & 3 & 1.1 & 1.7 & 2.2 & 1 & 2.2 & 1.1 & 2.0 & 1.8 & 1.2 & 1.5 & 3.2 & 2.8 & 0.7 & 0.7 & 2.5 & 0.4 \\
\hline V & 98.00 & 53 & 158 & 194 & 126 & 88 & 93 & 66 & 84 & 59 & 139 & 118 & 57 & 68 & 64 & 84 & 36 & 55 & 82 & 24 \\
\hline W & $<0.50$ & $<0.5$ & $<0.5$ & $<0.5$ & $<0.5$ & $<0.5$ & $<0.5$ & $<0.5$ & $<0.5$ & $<0.5$ & $<0.5$ & 0.5 & $<0.5$ & 0.6 & $<0.5$ & 0.6 & 1.4 & $<0.5$ & $<0.5$ & $<0.5$ \\
\hline $\mathrm{Zr}$ & 643.8 & 32.2 & 558.8 & 194.8 & 178 & 491 & 713 & 78.9 & 662 & 289 & 513 & 424 & 386 & 462 & 1087 & 1076 & 98.4 & 135.6 & 757.2 & 58.9 \\
\hline $\mathrm{La}$ & 36.00 & 24.9 & 46.8 & 64 & 35.6 & 39.7 & 53 & 24 & 43.7 & 28.7 & 40.3 & 46.5 & 25.8 & 29.9 & 45.6 & 46.1 & 22.7 & 14.9 & 53.2 & 4.8 \\
\hline $\mathrm{Ce}$ & 81.3 & 45.6 & 108.6 & 141.7 & 90.1 & 93.1 & 123 & 56.4 & 103 & 66.4 & 101 & 116 & 58.0 & 66.7 & 102 & 103 & 48.7 & 34.1 & 122.6 & 10 \\
\hline $\mathrm{Pr}$ & 9.51 & 6.87 & 12.36 & 16.36 & 9.98 & 10.4 & 13.5 & 6.4 & 11.2 & 7.12 & 11.0 & 12.7 & 6.34 & 7.41 & 11.3 & 11.6 & 5.33 & 3.98 & 13.6 & 1.14 \\
\hline $\mathrm{Nd}$ & 40.00 & 29.4 & 50.1 & 66.6 & 36.7 & 38.7 & 51.4 & 27.2 & 42.3 & 25.3 & 42.7 & 48.9 & 23.5 & 30.1 & 44.0 & 43.2 & 24.1 & 16.5 & 57 & 4.5 \\
\hline $\mathrm{Sm}$ & 6.83 & 5.64 & 9.53 & 13.15 & 7.31 & 7.28 & 9.68 & 4.85 & 8.50 & 5.22 & 8.35 & 10.2 & 3.87 & 5.02 & 8.74 & 8.91 & 4.09 & 3.07 & 11.43 & 0.92 \\
\hline $\mathrm{Eu}$ & 43 & 0.96 & 179 & 2.26 & 1.3 & 1.34 & 1.86 & 0.93 & 1.57 & 0.87 & 1.47 & 1.92 & 0.60 & 0.79 & 1.52 & 1.56 & 0.86 & 0.49 & 1.79 & 0.26 \\
\hline $\mathrm{Gd}$ & 00 & 4.79 & 7.74 & 12.47 & 5.69 & 5.67 & 7.9 & 4.24 & 6.51 & 4.54 & 6.65 & 8.99 & 3.25 & 4.35 & 7.67 & 7.53 & 3.69 & 2.24 & 9.11 & 0.86 \\
\hline $\mathrm{Tb}$ & 0.75 & 0.71 & 1.24 & 1.9 & 0.87 & 0.88 & 1.18 & 0.66 & 0.97 & 0.70 & 0.96 & 1.30 & 0.46 & 0.67 & 1.12 & 1.10 & 0.57 & 0.33 & 1.44 & 0.15 \\
\hline Dy & 02 & 3.63 & 71 & 10.44 & 3 & 4.21 & 6.11 & 3.68 & 4.97 & 3.35 & 4.78 & 5.96 & 2.34 & 3.28 & 5.20 & 5.26 & 2.52 & 1.62 & 7.02 & .03 \\
\hline $\mathrm{Y}$ & 18.6 & 20.4 & 20.0 & 52.4 & 17.7 & 19.4 & 26.8 & 17.1 & 24.3 & 18.2 & 20.3 & 32.5 & 11.9 & 18.9 & 28.3 & 27.0 & 17.0 & 7.6 & 36.2 & .7 \\
\hline Ho & 68 & 0.67 & 14 & 1.86 & 0.78 & 0.8 & 1.09 & 0.63 & 0.97 & 0.71 & 0.97 & 1.36 & 0.43 & 0.71 & 1.08 & 1.04 & 0.63 & 0.3 & 1.39 & 0.18 \\
\hline Er & 1.89 & 1.80 & 3.15 & 5.14 & 1.94 & 1.94 & . & 1.8 & 2.55 & 1.91 & 2.39 & 3.41 & 1.21 & 1.92 & 2.96 & 2.95 & 1.59 & 0.79 & 3.54 & 0.49 \\
\hline $\mathrm{Tm}$ & 0.29 & 0.26 & 0.49 & 0.73 & 0.27 & 0.31 & 0.42 & 0.25 & 0.39 & 0.27 & 0.36 & 0.44 & 0.19 & 0.29 & 0.46 & 0.43 & 0.26 & 0.11 & 0.55 & 0.08 \\
\hline $\mathrm{Yb}$ & 2.15 & 1.42 & 2.94 & 3.82 & 1.42 & 1.75 & 2.52 & 1.44 & 2.54 & 1.36 & 2.16 & 2.96 & 1.18 & 1.98 & 2.86 & 2.85 & 1.75 & 0.82 & 3.46 & 0.55 \\
\hline $\mathrm{Lu}$ & 0.29 & 0.20 & 0.41 & 0.57 & 0.22 & 0.3 & 0.38 & 0.2 & 0.38 & 0.22 & 0.32 & 0.47 & 0.18 & 0.29 & 0.47 & 0.42 & 0.22 & 0.1 & 0.46 & 0.07 \\
\hline Total & 209 & 147 & 281 & 393 & 214 & 226 & 302 & 150 & 254 & 165 & 244 & 293 & 139 & 172 & 264 & 263 & 134 & 87.0 & 323 & 29.7 \\
\hline
\end{tabular}


F. N. Kwankam et al.

Table 2. Major elements (wt\%), trace and rare-earth elements concentrations (ppm) of shales, calcareous shales, sandstones and lateritic soil.

\begin{tabular}{|c|c|c|c|c|c|c|c|c|c|c|c|c|c|c|c|c|c|c|c|c|}
\hline \multirow{2}{*}{$\begin{array}{c}\text { Rock } \\
\text { sample } \\
\text { No. }\end{array}$} & \multicolumn{9}{|c|}{ Shales } & \multicolumn{5}{|c|}{ Calcareous shales } & \multicolumn{5}{|c|}{ Sandstones } & \multirow{2}{*}{$\begin{array}{c}\text { Lt Soil } \\
\text { S8 }\end{array}$} \\
\hline & S5 & S19 & S23 & S25 & S29 & S30 & S31 & S33 & S39 & S11 & S15 & S17 & S21 & S38 & S2 & S9 & S12 & S28 & S32 & \\
\hline $\mathrm{SiO}_{2}$ & 50.57 & 61.77 & 51.90 & 58.09 & 69.9 & 72.2 & 70.5 & 51.1 & 47.6 & 47.2 & 49.3 & 29.0 & 30.6 & 44.6 & 84 & 85.2 & 72.5 & 53.17 & 94.37 & 28.9 \\
\hline $\mathrm{TiO}_{2}$ & 2.21 & 0.90 & 1.94 & 3.04 & 2.40 & 2.88 & 0.77 & 2.50 & 2.22 & 1.55 & 2.15 & 1.0 & 1.09 & 1.70 & 0.5 & 1.59 & 0.69 & 0.27 & 0.20 & 1.2 \\
\hline $\mathrm{Al}_{2} \mathrm{O}_{3}$ & 18.89 & 7.68 & 15.05 & 14.90 & 11.3 & 12.1 & 11.6 & 18.9 & 17.6 & 17.2 & 16.3 & 6.42 & 6.77 & 15.8 & 3.7 & 8.37 & 11.1 & 2.12 & 3.68 & 12.32 \\
\hline $\mathrm{Fe}_{2} \mathrm{O}_{3}$ & 5.39 & 13.72 & 11.40 & 5.65 & 3.68 & 5.31 & 2.94 & 6.49 & 10.3 & 10.4 & 11.6 & 7.62 & 6.76 & 17.4 & 8.3 & 0.43 & 2.38 & 36.81 & 0.17 & 43.43 \\
\hline $\mathrm{MnO}$ & 0.02 & 0.05 & 0.07 & 0.03 & 0.02 & $<0.01$ & 0.02 & 0.03 & 0.03 & 0.05 & 0.05 & 0.08 & 0.08 & 0.05 & 0.01 & $<0.01$ & $<0.01$ & $<0.01$ & $<0.01$ & 0.10 \\
\hline $\mathrm{MgO}$ & 0.91 & 1.94 & 1.53 & 0.43 & 0.10 & 0.09 & 0.40 & 0.50 & 1.23 & 1.52 & 1.57 & 1.45 & 1.30 & 2.36 & 0.02 & 0.06 & 0.4 & $<0.01$ & $<0.01$ & 0.09 \\
\hline $\mathrm{CaO}$ & 0.24 & 2.75 & 0.50 & 0.13 & 0.03 & $<0.01$ & 0.06 & 0.07 & 0.65 & 3.98 & 0.97 & 27.5 & 26.5 & 1.53 & 0.14 & 0.21 & 0.69 & 0.05 & $<0.01$ & 0.44 \\
\hline $\mathrm{Na}_{2} \mathrm{O}$ & 0.38 & 0.16 & 0.45 & 0.56 & 0.07 & 0.03 & 0.10 & 0.08 & 0.24 & 0.27 & 0.45 & 0.28 & 0.28 & 0.17 & 0.02 & 0.02 & 0.29 & $<0.01$ & $<0.01$ & $<0.01$ \\
\hline $\mathrm{K}_{2} \mathrm{O}$ & 2.04 & 0.90 & 2.76 & 3.15 & 1.78 & 0.54 & 2.57 & 2.26 & 2.28 & 2.11 & 2.68 & 1.8 & 1.84 & 1.53 & 0.03 & 0.17 & 2.29 & 0.01 & 0.02 & 0.1 \\
\hline $\mathrm{P}_{2} \mathrm{O}_{5}$ & 0.08 & 1.24 & 0.08 & 0.06 & 0.14 & 0.12 & 0.04 & 0.08 & 0.08 & 0.10 & 0.06 & 0.07 & 0.06 & 0.08 & 0.05 & $<0.01$ & 0.06 & 0.26 & 0.02 & 0.59 \\
\hline $\mathrm{Cr}_{2} \mathrm{O}_{3}$ & 0.01 & 0.04 & 0.02 & 0.021 & 0.02 & 0.02 & 0.01 & 0.02 & 0.02 & 0.02 & 0.02 & 0.01 & 0.01 & 0.02 & 0.01 & 0.01 & 0.01 & 0.01 & $<0.002$ & 0.07 \\
\hline LOI & 19.00 & 8.50 & 13.90 & 13.6 & 10.1 & 6.4 & 10.7 & 17.6 & 17.6 & 15.3 & 14.6 & 24.4 & 24.4 & 14.5 & 2.8 & 3.8 & 9.4 & 7.3 & 1.6 & 12.5 \\
\hline Total & 99.743 & 99.646 & 99.60 & 99.66 & 99.5 & 99.7 & 99.7 & 99.6 & 99.8 & 99.7 & 99.7 & 99.6 & 99.7 & 99.7 & 99.9 & 99.8 & 99.8 & 99.99 & 100.06 & 99.74 \\
\hline $\mathrm{Ni}$ & 34 & 25 & 57 & 27 & $<20$ & $<20$ & $<20$ & 48 & 67 & 57 & 37 & $<20$ & $<20$ & 80 & $<20$ & $<20$ & $<20$ & $<20$ & $<20$ & 32 \\
\hline $\mathrm{Sc}$ & 13 & 12 & 13 & 13 & 9 & 10 & 6 & 13 & 15 & 13 & 14 & 8 & 7 & 16 & 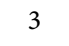 & 5 & 5 & 7 & $<1$ & 20 \\
\hline $\mathrm{Ba}$ & 688 & 283 & 809 & 812 & 1378 & 693 & 1142 & 897 & 593 & 681 & 743 & 527 & 558 & 578 & 26 & 88 & 662 & 7 & 26 & 65 \\
\hline $\mathrm{Be}$ & 3 & 2 & 4 & 3 & 2 & 2 & 1 & 4 & 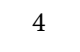 & 4 & $<1$ & 1 & 1 & $<1$ & 2 & $<1$ & $<1$ & 1 & 1 & 7 \\
\hline Co & 12.4 & 20.8 & 48.1 & 9.4 & 12.0 & 1.8 & 8.5 & 24.3 & 29.2 & 30.2 & 33.4 & 20.8 & 20.4 & 40.0 & 2.7 & 0.5 & 10.3 & 0.5 & 0.3 & 29.3 \\
\hline Cs & 1.9 & 0.7 & 1.4 & 1.0 & 0.7 & 0.3 & 0.5 & 1.9 & 1.3 & 1.8 & 1.8 & 0.6 & 0.6 & 1.6 & 0.1 & $<0.1$ & 0.9 & $<0.1$ & $<0.1$ & 0.6 \\
\hline $\mathrm{Ga}$ & 28.6 & 13.1 & 24.8 & 25.6 & 14.8 & 16.2 & 12.5 & 27.6 & 23.7 & 24.6 & 24.4 & 9.7 & 10.0 & 23.3 & 3.4 & 8.2 & 13.4 & 3.8 & 3.1 & 19.4 \\
\hline $\mathrm{Hf}$ & 18.4 & 12.5 & 20.7 & 25.9 & 46.4 & 39.6 & 20.5 & 31.4 & 17.5 & 11.6 & 15.4 & 21.1 & 22.7 & 13.5 & 2.9 & 26.4 & 6.3 & 4.1 & 3.1 & 21.2 \\
\hline $\mathrm{Nb}$ & 65.6 & 29.6 & 54.0 & 71.4 & 53.7 & 61.3 & 21.4 & 89.3 & 48.5 & 43.4 & 45.5 & 26 & 35.9 & 40.5 & 13 & 32.5 & 18.9 & 7.1 & 6.1 & 31.7 \\
\hline $\mathrm{Rb}$ & 57.9 & 24.6 & 69.6 & 69.0 & 42.3 & 16.1 & 48.3 & 58.9 & 55.0 & 55.2 & 63.4 & 39 & 38.3 & 38.0 & 1.5 & 4.9 & 48.9 & 0.9 & 0.5 & 7.3 \\
\hline Sn & 6 & 3 & 4 & 7 & 3 & 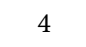 & $<1$ & 7 & 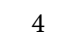 & 5 & 6 & 2 & 2 & 1 & $<1$ & 2 & 2 & $<1$ & 1 & 3 \\
\hline $\mathrm{Sr}$ & 96.6 & 160.7 & 139.3 & 108.8 & 206 & 128 & 162 & 136 & 121 & 269 & 149 & 838 & 737 & 141 & 13 & 13.7 & 107 & 6.3 & 4.0 & 30.9 \\
\hline $\mathrm{Ta}$ & 4.4 & 4.3 & 3.2 & 3.8 & 3.2 & 3.8 & 1.0 & 5.9 & 2.7 & 7.5 & 2.2 & 1.4 & 2.3 & 2.6 & 1 & 3.5 & 2.3 & 0.5 & 0.4 & 2.2 \\
\hline Th & 19.5 & 18 & 18.3 & 20.2 & 20.7 & 21.4 & 10.8 & 24.6 & 19.2 & 17.5 & 17.8 & 12 & 11.5 & 16.9 & 4.9 & 11.1 & 7.4 & 3.4 & 1.3 & 19.1 \\
\hline $\mathrm{U}$ & 4.6 & 6.5 & 3.5 & 4.4 & 6.7 & 6.1 & 2.9 & 6.5 & 4.4 & 3.3 & 3.5 & 2.7 & 3.0 & 3.5 & 0.9 & 2.1 & 1.4 & 3.2 & 0.4 & 8.6 \\
\hline V & 109 & 399 & 181 & 157 & 96 & 119 & 41 & 121 & 144 & 228 & 189 & 105 & 90 & 182 & 27 & 57 & 82 & 37 & 9 & 524 \\
\hline W & 1.3 & $<0.5$ & 1.5 & 1.9 & 1.2 & 1.0 & 0.7 & 1.4 & 1.2 & 1.2 & 1.2 & $<0.5$ & 1.3 & 1.7 & $<0.5$ & 1.5 & 0.8 & $<0.5$ & $<0.5$ & 0.9 \\
\hline $\mathrm{Zr}$ & 703.7 & 470.9 & 772.2 & 972.6 & 1746 & 1425 & 801 & 1114 & 649 & 447 & 565 & 794 & 830 & 537 & 98.9 & 1012 & 227.8 & 181.2 & 136.9 & 843.5 \\
\hline $\mathrm{La}$ & 76.50 & 95.40 & 77.30 & 66.20 & 45.1 & 41.9 & 29.0 & 79.5 & 67.3 & 66.9 & 60.7 & 64.1 & 53.5 & 77.8 & 9.6 & 12.4 & 56.7 & 7.7 & 2.2 & 36 \\
\hline $\mathrm{Ce}$ & 156.1 & 231.10 & 177.50 & 151.0 & 94.3 & 84.0 & 60.4 & 170.8 & 137.8 & 145.3 & 136.4 & 145.9 & 122.9 & 165.7 & 46 & 24.2 & 101 & 14.2 & 5.0 & 110.8 \\
\hline $\operatorname{Pr}$ & 18.41 & 26.14 & 19.69 & 16.33 & 10.1 & 9.09 & 6.77 & 18.49 & 15.82 & 17.28 & 15.95 & 16.4 & 13.16 & 19.32 & 1.9 & 2.84 & 13.8 & 1.56 & 0.45 & 7.71 \\
\hline
\end{tabular}




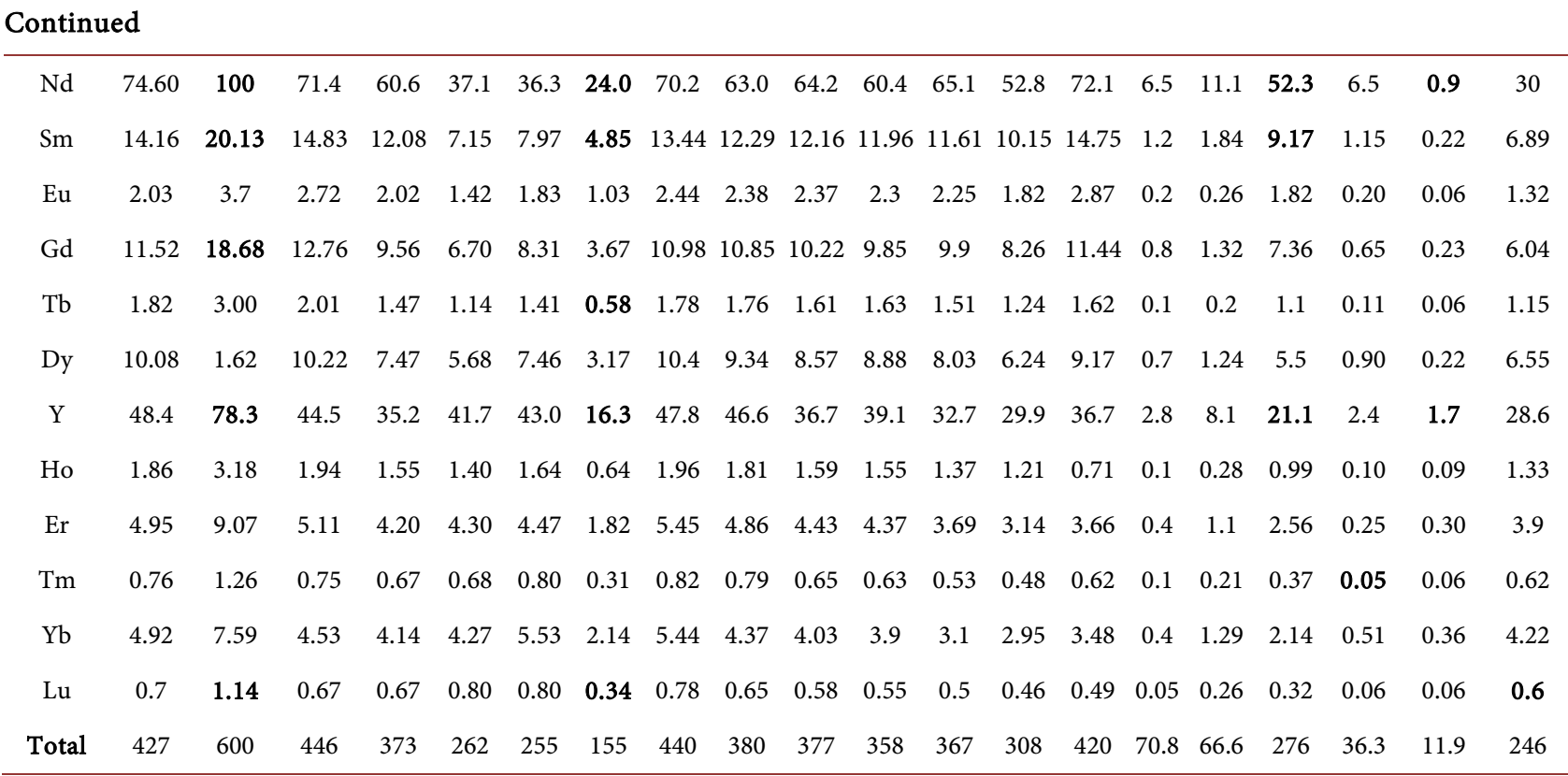

\subsection{Major Elements}

The concentrations of the major oxides in the carbonates, shales and sandstones are presented in Tables 1 and Table 2 respectively. The geochemical data show low to moderate concentrations for $\mathrm{SiO}_{2}$ in the limestones (2.43-35.5 wt\%) and moderate to high concentrations for marly limestones (37.46 - $54.31 \mathrm{wt} \%$ ).

The major oxides also show moderate to very high values of $\mathrm{SiO}_{2}(47.6-72.26$ wt\%) for shale and moderate $\mathrm{SiO}_{2}$ values $(29.0$ - $49.3 \mathrm{wt} \%)$ for calcareous shale (Table 2). Sandstones from the study area have the highest $\mathrm{SiO}_{2}$ concentration (53.17 - $94.37 \mathrm{wt} \%$; Table 2). The single lateritic soil sample gave a moderate $\mathrm{SiO}_{2}$ value (28.9 wt\%; Table 2).

The concentration of $\mathrm{CaO}$ in limestone ranges from 19.32 to $51.52 \mathrm{wt} \%$ and 19.5 to $23.19 \mathrm{wt} \%$ in marly limestone (Table 1 ). $\mathrm{CaO}$ concerntration for the carbonate lithofacies are generally high. $\mathrm{CaO}$ concentrations in shales are low $(<0.01-2.75 \mathrm{wt} \%)$ and low to moderate $(0.97-27.5 \mathrm{wt} \%)$ for calcareous shales (Table 2). The concentration of $\mathrm{CaO}$ for sandstones and the lateritic soil sample are significantly low $(<0.01-0.69 \mathrm{wt} \%)$. From the major oxides results presented on Table 2, it is observed that samples with higher $\mathrm{SiO}_{2}$ concentrations have lower $\mathrm{CaO}$ content. However, the $\mathrm{CaO}$ content for a marly limestone sample (S13) is significantly high (19.5 wt\%) when compared to its high $\mathrm{SiO}_{2}$ content (54.31 wt\%). For carbonates, high $\mathrm{CaO}$ content of $\geq 50 \mathrm{wt} \%$ signifies medium to high purity [43]. Moderate concentration of $\mathrm{CaO}$ between 26.5 to $27.5 \mathrm{wt} \%$ (Table 2), obtained for some calcareous shales is indicative of biogenic/organic input which precipitates $\mathrm{CaO}$. Aluminium oxide $\left(\mathrm{Al}_{2} \mathrm{O}_{3}\right)$ concentration is slightly lower in limestones (0.98 - $8.09 \mathrm{wt} \%)$ compared to marly limestones (3.09 - 7.95 wt\%) (Table 1). The shales and calcareous shales show $\mathrm{Al}_{2} \mathrm{O}_{3}$ concentrations ranging from 6.42 to $18.9 \mathrm{wt} \%$, while those for sandstones vary between 2.12 $11.1 \mathrm{wt} \%$ (Table 2). Iron oxide $\left(\mathrm{Fe}_{2} \mathrm{O}_{3}\right)$ concentration in limestones ranges from 
2.17 to 10.68 wt\% with slightly lower values in marly limestones (3.09 - 6.70 wt\%) and slightly higher values (2.94 - $13.72 \mathrm{wt} \%)$ in shales and calcareous shales $(6.76$ - $17.4 \mathrm{wt} \%)$. Iron oxide $\left(\mathrm{Fe}_{2} \mathrm{O}_{3}\right)$ concentrations range from 2.94 13.72 , wt $\%)(6.76-17.4,0.17-36.81 \mathrm{wt} \%)$ and $43.43 \mathrm{wt} \%$ for the shales, calcareous shales, sandstones and laterite soil sample, respectively. The concentrations of titanium oxide $\left(\mathrm{TiO}_{2}\right)$ manganese oxide $(\mathrm{MnO})$, phosphorus pentaoxide $\left(\mathrm{P}_{2} \mathrm{O}_{5}\right)$ and chromium dioxide $\left(\mathrm{Cr}_{2} \mathrm{O}_{3}\right)$ have significantly low values in both limestones and marly limestones with slightly higher values for $\mathrm{MgO}$ and $\mathrm{K}_{2} \mathrm{O}$ (Table 1).

\subsection{Trace Elements}

Trace elements are very vital in the identification of geological processes like provenance and tectonic settings. These elements were normalized using North American shale composite (NASC) values from [44]. Normalization against NASC is a measure of subtle enrichment and deficiencies in certain elements in the sediments [5]. The carbonate rocks (limestones and marly limestones) show low to moderate concentrations of $\mathrm{Ni}$, Co, Be, Hf, Nb, Y, W, Sn and Th (<0.5 - 40 ppm; Table 1). Lower concentrations of $\mathrm{Cs}, \mathrm{Ga}, \mathrm{Sc}, \mathrm{Ta}, \mathrm{U}(<0.1-12.7 \mathrm{ppm})$ are also observed, with the highest values obtained for $\mathrm{Ba}, \mathrm{Rb}, \mathrm{Sn}, \mathrm{Sr}, \mathrm{Zr}$ and $\mathrm{V}(<1$ $1456 \mathrm{ppm})$ in the rocks. The shale and calcareous shale samples display low concentrations of $\mathrm{Be}, \mathrm{Sn}, \mathrm{Ta}, \mathrm{U}$ and $\mathrm{W}(<0.5-7.5 \mathrm{ppm})$, low to moderate concentrations of Sc, Co, Ga and Hf (6 - $48.1 \mathrm{ppm})$, moderate to high concentrations of $\mathrm{Ni}, \mathrm{Th}, \mathrm{Nb}$ and $\mathrm{Rb}(10.8-89.3 \mathrm{ppm})$ and high to very high concentrations for Ba, Sr, V and Zr (41 - 1746 ppm) (Table 2). The sandstones are characterized by low concentrations of $\mathrm{Be}, \mathrm{Cs}, \mathrm{Sn}, \mathrm{Ta}, \mathrm{U}$ and $\mathrm{W}(<0.1-3.5 \mathrm{ppm})$, moderate concentrations of $\mathrm{Ni}, \mathrm{Co}, \mathrm{Sc}, \mathrm{Ga}, \mathrm{Hf}, \mathrm{Nb}, \mathrm{Rb}, \mathrm{Sr}$ and $\mathrm{Th}(<1-107$ ppm) and high concentrations of $\mathrm{Ba}, \mathrm{V}$ and $\mathrm{Zr}$ (7 - $1012 \mathrm{ppm}$ ).

From the studied lithofacies, the trace element compositions of the carbonate rocks, calcareous shale and shales and sandstones were normalized against the North American shale Composite (NASC) [44]. The carbonates show depletion of Sc, Cs, Rb, Ta and Th with respect to those of NASC; with enrichment of Hf, $\mathrm{Sr}$, and $\mathrm{Zr}$ and moderate depletion of $\mathrm{Ni}, \mathrm{Ba}, \mathrm{Co}$ and $\mathrm{U}$ when compared to NASC. Regular patterns were observed for $\mathrm{Ca}, \mathrm{Cs}, \mathrm{Hf}, \mathrm{Rb}$ to $\mathrm{Sr}$, with scattered plots observed for Ni, Sc, Ba, Ta, Th, U and Zr (Figure 1). Based on the normalized trace elements data, shales and calcareous shales exhibit severe depletion in Co and $\mathrm{Cs}$, moderate depletion in $\mathrm{Ni}, \mathrm{Sc}$ and $\mathrm{Rb}$, and enrichment in $\mathrm{Ba}, \mathrm{Hf}, \mathrm{Rb}$, $\mathrm{Sr}, \mathrm{Ta}, \mathrm{Th}, \mathrm{U}$ and $\mathrm{Zr}$ relative to the NASC (Figure 4(a)). Relatedly, the normalized sandstones data display significant variations including enrichment in Sc, $\mathrm{Hf}$, Ta, Th, $\mathrm{U}$ and $\mathrm{Zr}$, moderate depletion in $\mathrm{Ni}$ and $\mathrm{Sr}$, and severe depletion in $\mathrm{Ba}, \mathrm{Co}, \mathrm{Cs}$ and $\mathrm{Rb}$ compared to the NASC (Figure 4(b)). Overall, data for the samples for elements that are abundant in ferromagnesian minerals (e.g., Co and $\mathrm{Ni}$ ) and high field strength elements (Zr, Hf, Th and $\mathrm{U}$ ) show depletion relative to the NASC. 

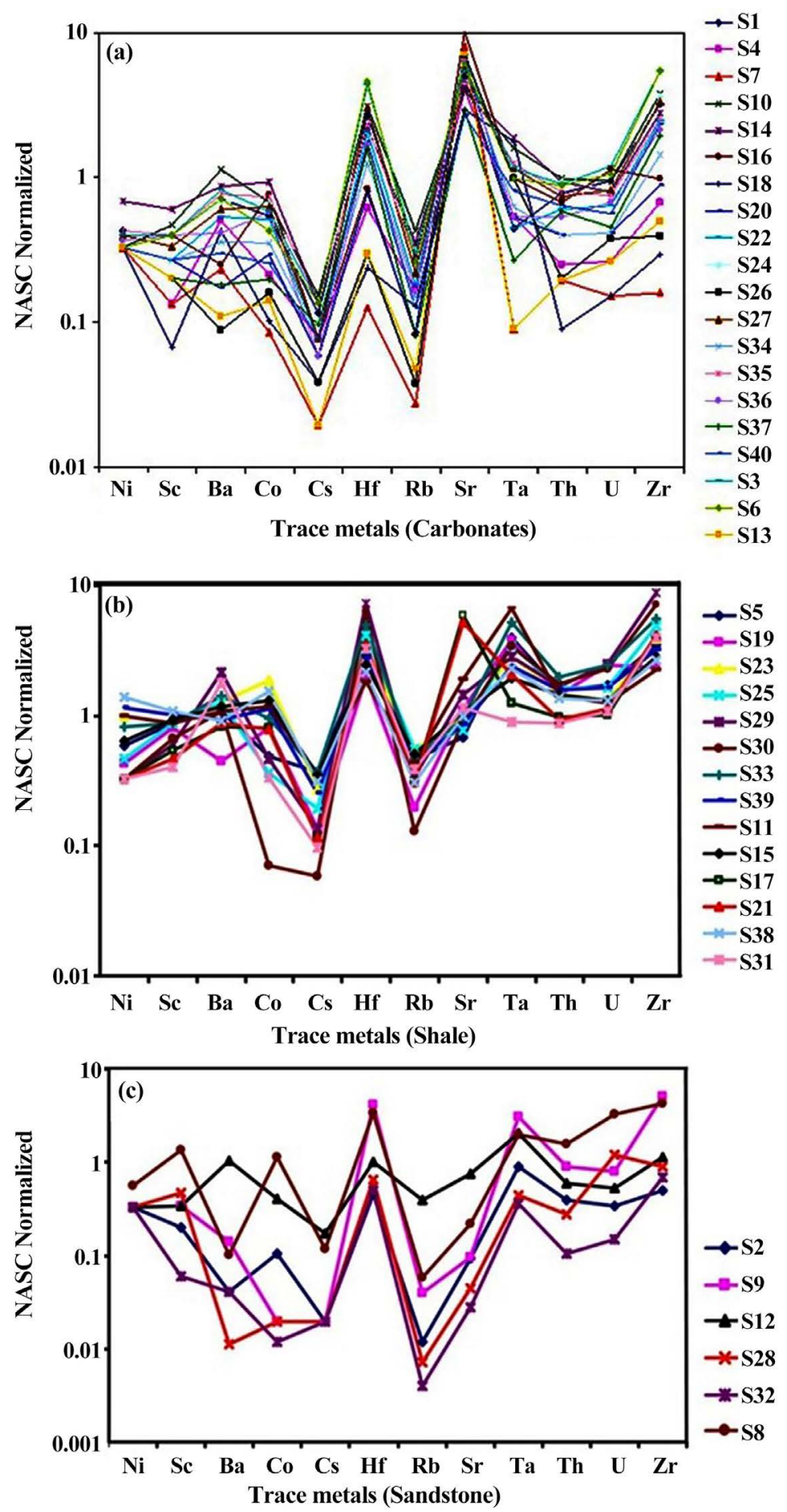

Figure 4. North American shale composite (NASC)-normalised trace element diagrams for (a) carbonate rocks, (b) shales and (c) sandstones of the Nkapa Formation. The NASC values of [44] were used, and these plots are useful in identifying subtle enrichment and deficiencies in certain elements [5].

\subsection{Rare Earth Elements (REEs)}

The REE data for the various lithofacies studied are given on Table 1 and Table 
2. The highest concentration of REEs in the limestones and marly limestones was obtained for La and Ce, representing light rare elements (LREE) with values ranging from 4.8 to $64 \mathrm{ppm}$ and $10-141.7 \mathrm{ppm}$ respectively. High concentrations were also obtained for $\mathrm{Nd}(4.5-66.6 \mathrm{ppm})$ and $\mathrm{Y}(4.7-52.4 \mathrm{ppm})$. Middle REEs which range from $\mathrm{Sm}$ to Ho have low $(0.15 \mathrm{ppm}$; $\mathrm{Tb})$ to moderate $(13.15$ ppm; Sm) concentrations with the lowest values obtained from the heavy rare earth elements (Er to Lu; HREE) e.g., Lu with concentration of 0.07 to $0.47 \mathrm{ppm}$ (Table 1). The REEs in the limestones and marly limestones have mean values ranging from $0.31 \mathrm{ppm}(\mathrm{Lu})$ to $83.63 \mathrm{ppm}$ (Ce; Table 1 ).

Among the shale and calcareous shale samples analyzed, Ce displays the highest concentrations (60.4 - $231.10 \mathrm{ppm}$ ) among the LREEs, followed by Nd (24 to $100 \mathrm{ppm})$, La (29.0 - 95.40 ppm) and Y (16.3 - 78.3 ppm). For the MREEs, Sm has a concentration of 4.85 to $20.13 \mathrm{ppm}, \mathrm{Eu}, \mathrm{Gd}, \mathrm{Tb}, \mathrm{Dy}$, Ho had concentrations in the range of $0.58-18.68 \mathrm{ppm}$ (Table 2). In the shale samples, same as for the carbonates, the lowest concentration for the REE was obtained for the HREE (Lu) with concentrations of $0.34-1.14 \mathrm{ppm}$. For shale samples mean REE values are between $0.7(\mathrm{Lu})$ - 141.4 (Ce) ppm (Table 2). Regarding the sandstones, Ce $(5.0$ - $101 \mathrm{ppm})$ also showed the highest concentrations among LREEs, with subordinate concentrations for La (2.2 - $56.7 \mathrm{ppm})$, Nd (0.9 - 52.3 ppm), Pr (0.45 - $13.8 \mathrm{ppm})$ and Y (1.7 - 21.1 ppm). Both the MREE and HREEs (Sm, Eu, Gd, Tb, Dy, Ho, Er, Tm, Yb and Lu) are characterised by low concentrations varying correspondingly between $0.05-9.17$ and 0.2 to $50.2 \mathrm{ppm}$ (Table 2).

\subsection{Element Ratios}

The studied samples are characterized by $\mathrm{V} /(\mathrm{V}+\mathrm{Ni})$ ratios varying between 0.56 - 0.87 for the carbonates, 0.69 - 0.94 for calcareous shales and $0.32-0.94$ for sandstones (Table 3).

The La/Co ratios vary from 1.94 - 11.32, 1.85 - 3.88, $3.56-24.80$ and 1.61 23.28 for the limestones, marly limestones, sandstones and, shales and calcareous shales respectively (Table 4). The La/Co ratio of 24.80 for sample S9 is significantly high among the sandstone samples, while a shale sample (S30) shows a ratio beyond the maximum reported for felsic rocks (Table 4).

\section{Discussion}

\subsection{Provenance Indicators}

Trace elements are vital indicators of geological processes, such as provenance and tectonic settings associated with the deposition of sediments. The high REE concentrations in the shale samples are probably caused by terrigenous input. The low to moderate REE concentrations in the sandstones are attributed to quartz dilution [45] [46]. The Eu/Eu*, La/Sc, La/Co, Th/Co and Th/Cr ratios are generally employed as indicators of the provenance of sediments (Table 3: [45] [46] [47]). 
F. N. Kwankam et al.

Table 3. Element ratios in limestones, marly limestones, shales, calcareous shales, sandstones and lateritic soil.

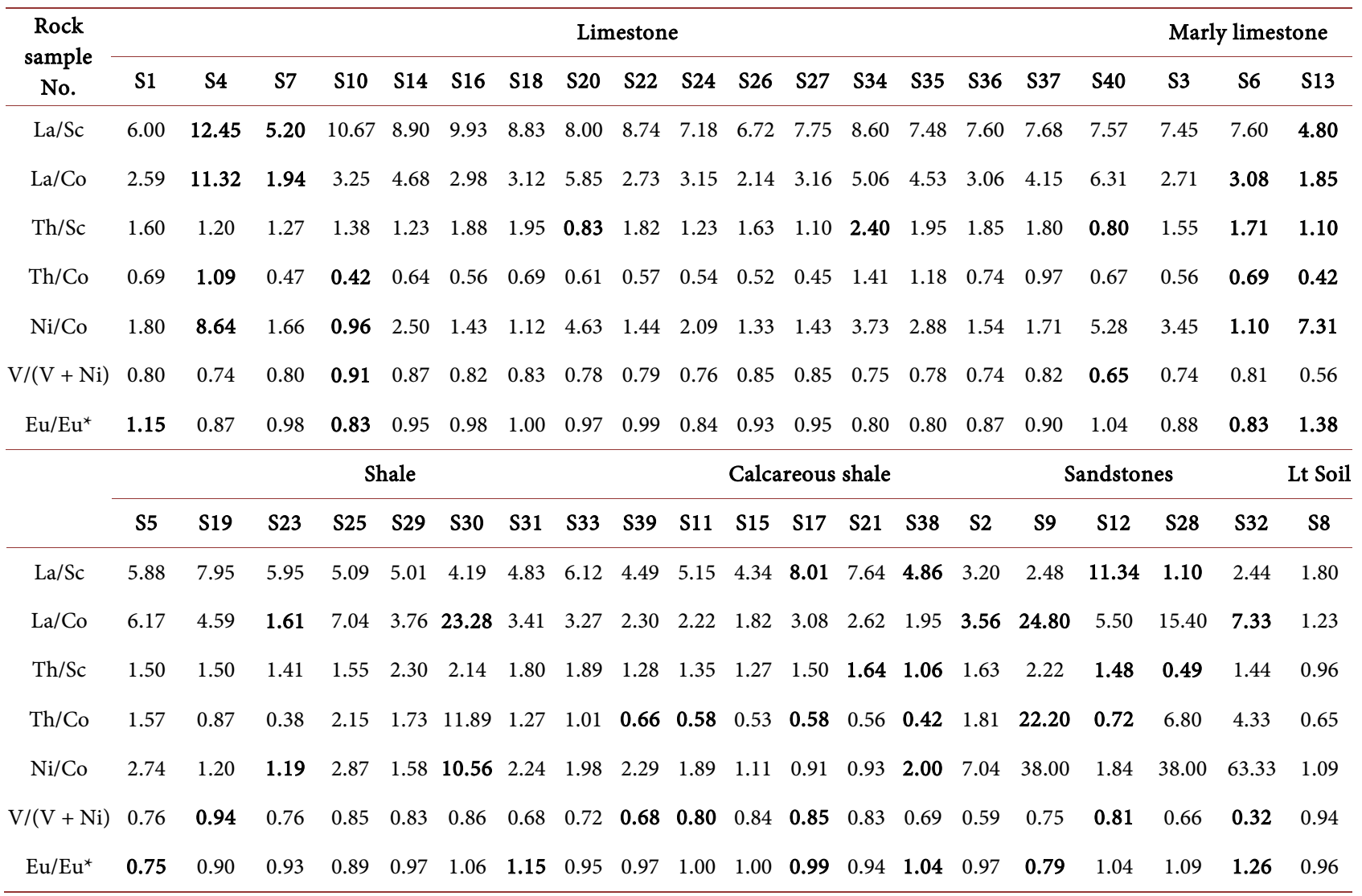

NB: Lt = Lateritic soil.

Table 4. Range of element ratios for the different lithofacies of the study area compared to felsic rocks and mafic rocks.

\begin{tabular}{|c|c|c|c|c|c|c|c|c|}
\hline \multirow[t]{2}{*}{ Rock } & \multicolumn{2}{|c|}{ Limestone } & \multicolumn{2}{|r|}{ Shale } & \multicolumn{2}{|c|}{ Terrigenous sediments } & \multicolumn{2}{|c|}{ Ranges for felsic and mafic rocks } \\
\hline & Limestone & Marly limestone & Shale & Calcareous Shale & Sandstone & Lt. Soil & Felsic rocks & Mafic rocks \\
\hline $\mathrm{La} / \mathrm{Sc}$ & $5.20-12.45$ & $4.80-7.96$ & $4.19-7.95$ & $4.34-8.01$ & $1.10-11.34$ & 1.80 & $2.50-16.3$ & $0.43-0.86$ \\
\hline $\mathrm{La} / \mathrm{Co}$ & $1.94-11.32$ & $1.85-3.88$ & $1.61-23.28$ & $1.82-3.08$ & $3.56-24.80$ & 1.23 & $1.80-13.8$ & $0.14-0.38$ \\
\hline $\mathrm{Th} / \mathrm{Sc}$ & $0.80-2.40$ & $1.10-1.71$ & $4.86-8.01$ & $1.06-1.64$ & $0.49-1.48$ & 0.96 & $0.84-20.5$ & $0.05-0.22$ \\
\hline $\mathrm{Th} / \mathrm{Co}$ & $0.42-1.09$ & $0.42-1.09$ & $0.66-11.89$ & $0.42-0.58$ & $0.72-22.20$ & 0.65 & $0.67-19.4$ & $0.04-1.40$ \\
\hline
\end{tabular}

NB: Mafic and felsic values were derived by [45] [46].

These ratios for the studied shales and sandstones were used to support the major element discriminants of felsic and mafic rocks in highlighting their provenance (Figure 5).

$\mathrm{The} \mathrm{Th} / \mathrm{Sc}$ ratios for the shales (4.86 - 8.01), calcareous shales (1.06 - 1.64) and sandstones $(0.49-1.48)$ are indicative of provenance from felsic sources (Table 4 ; [10]).

However, the significantly low $\mathrm{Th} / \mathrm{Sc}$ ratio of 0.49 for a sandstone sample (S28) falls outside the ranges for felsic and mafic rock sources (Table 3). The 


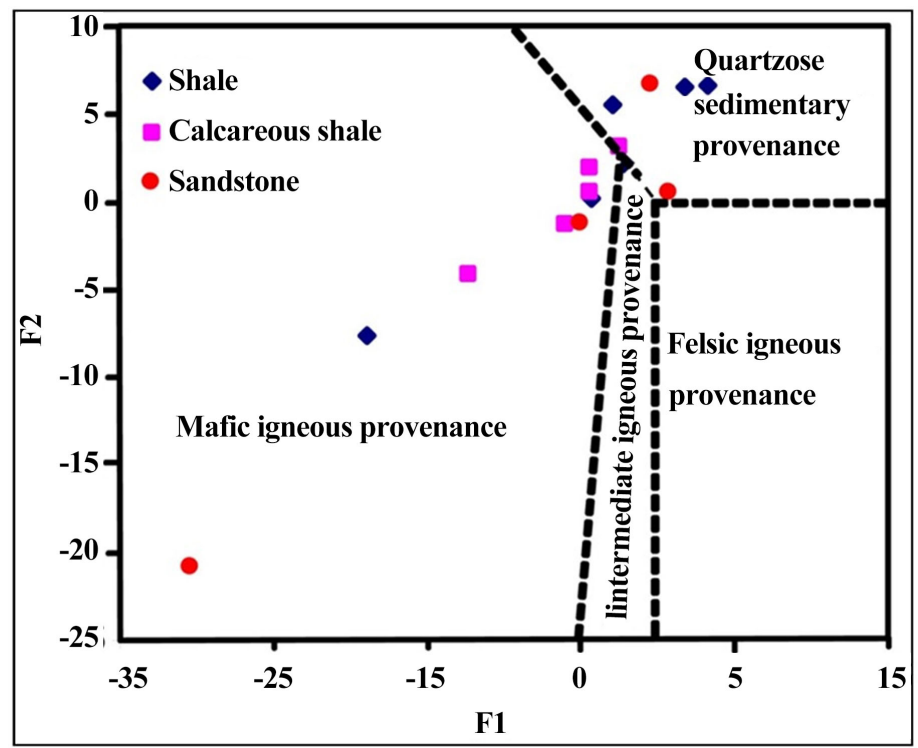

Figure 5. Discrimination diagram for the provenance of sedimentary rocks showing the (a) Kompina sandstones and shales after [10]. F1 = $30.638 \mathrm{TiO}_{2} / \mathrm{Al}_{2} \mathrm{O}_{3}-12.541 \mathrm{Fe}_{2} \mathrm{O}_{3}$ (total) $/ \mathrm{Al}_{2} \mathrm{O}_{3}+7.329 \mathrm{MgO} / \mathrm{Al}_{2} \mathrm{O}_{3}+$ $12.031 \mathrm{Na}_{2} \mathrm{O} / \mathrm{Al}_{2} \mathrm{O}_{3}+35.402 \mathrm{~K}_{2} \mathrm{O} / \mathrm{Al}_{2} \mathrm{O}_{3}-6.382 . \mathrm{F} 2=56.5 \mathrm{TiO}_{2} / \mathrm{Al}_{2} \mathrm{O}_{3}-$ $10.879 \mathrm{Fe}_{2} \mathrm{O}_{3}$ (total) $/ \mathrm{Al}_{2} \mathrm{O}_{3}+30.875 \mathrm{MgO} / \mathrm{Al}_{2} \mathrm{O}_{3}-5.404 \mathrm{Na}_{2} \mathrm{O} / \mathrm{Al}_{2} \mathrm{O}_{3}+$ $11.112 \mathrm{~K}_{2} \mathrm{O} / \mathrm{Al}_{2} \mathrm{O}_{3}-3.89$.

$\mathrm{Th} / \mathrm{Co}$ ratios for the shales $(0.66-11.89)$ and sandstones $(0.72-22.20)$ also reflect provenance dominantly from felsic sources. This is also supported by the $\mathrm{La} / \mathrm{Co}$ ratios for limestones, marly limestones, sandstones, shales and calcareous shales (Table 4). Based on comparisons of the trace and rare earth element ratios for the samples studied and those for felsic and mafic rocks proposed by [45] [46], almost all the shales and sandstones from the Nkapa Formation originated from felsic sources with subordinate inputs from other sources (Table 3). Notably, the $\mathrm{Eu}^{2+}$ anomaly is mainly controlled by feldspars from felsic magmas, in contrast to the incompatible $\mathrm{Eu}^{3+}$. According to data in Table 3 and in Figure 4(c), approximately two-thirds of the sandstone samples display positive Eu anomalies. The negative Eu anomalies observed for sandstones imply that these components were transported to the marine environment as part of detrital sand grains.

According to the oxides-based discrimination diagram proposed by [10], provenance fields include the mafic igneous, quartzose sedimentary and intermediate igneous (Figure 5). However, few shales and no sandstone from the Nkapa Formation plot in the intermediate igneous provenance field (Figure 5). In addition, no sample falls in the felsic igneous provenance field, which apparently contradicts the dominantly felsic origin for the studied samples based on trace element ratios (Table 4). However, these data highlight the presence of mafic plutons within a largely granitic (felsic) source region. This discrimination diagram further indicates that the sediments which were formed these rocks were derived from multiple sources, and this is consistent with the felsic, mafic, 
quartzose and minor intermediate provenance displayed in Figure 5. The Mafic igneous and felsic igneous provenances may have resulted from the faulted basement rocks which are made up of granite and Gneissic rocks in Cameroon and as well as from volcanic mafic rocks from the CVL and the Felsic Line [15] [16].

\subsection{Tectonic Setting Discrimination}

Elements including $\mathrm{La}, \mathrm{Ce}, \mathrm{Nd}, \mathrm{Y}, \mathrm{Th}, \mathrm{Hf}, \mathrm{Nb}, \mathrm{Ti}$, and $\mathrm{Sc}$ are suitable for provenance and tectonic discrimination [3] [4] because of their low mobilities during sedimentary processes and their low residence times in seawater [5]. These elements, which are transported with clastic sediments after weathering, commonly retain signatures of the parent materials [48] [49].

Triangular plots [4] were employed for the discrimination of tectonic settings associated with the rocks from the Nkapa Formation in the present study, as shown in Figure 6(a) and Figure 6(b)). The sandstones and shales plot mainly in the passive margin (PM) and continental island arc (CIA) fields, but some shale samples also fall in the ocean island arc (OIA) and active continental margin (ACM) fields. The remainder of the sandstones and shales fall out of the four demarcated tectonic zones in the diagrams and area could be adjacent areas such as oceanic and contininental rift zones. In Figure 6(a) and Figure 6(b), no sandstone sample from the Nkapa Formation plots in the ACM field.

According to plots involving major oxides (e.g., $\mathrm{TiO}_{2}$ vs. $\mathrm{Fe}_{2} \mathrm{O}_{3}+\mathrm{MgO}$ ), the sandstones studied mostly fall in the PM field, while others are outside the fields assigned to other settings (Figure 7). Regarding the shales, majority of the samples plot in the CIA and ACM fields, while few fall in the PM field (Figure 7), in contrast to the trace element triangular plots (Figure 6). The major oxide plots show in the OIA field (Figure 7).

According to the trace elements and major oxides plots, the sandstones in the Nkapa Formation are mainly associated with the PM and CIA settings, while the shales dominantly belong to PM, CIA and ACM settings (Figure 6(a) and Figure 6(b)). Using discrimination diagrams proposed in [10] [12] analyzed claystones and siltstones collected from the Cretaceous/Tertiary Nanxiong Basin in China for tectonic association (Figure 6(d)). The rocks from the Nanxiong Basin plot predominantly in the PM field (Figure 6(c), Figure 6(d)), similar to rocks in the present study, with some deviations (Figures 6(a)-(c)). The presence of sandstones dominantly in the PM field is attributed to their high quartz contents and the associated resistance to weathering during transportation from source area [10].

In addition, the $\mathrm{K}_{2} \mathrm{O} / \mathrm{Na}_{2} \mathrm{O}$ ratios of sandstones from the study area range from 1 to 8.5 . According to [10], sandstones with $\mathrm{K}_{2} \mathrm{O} / \mathrm{Na}_{2} \mathrm{O}>1$ are commonly quartz-rich, and their compositions are similar to those of arenites, which are dominantly deposits of PM and plate interior settings. These results supports the tectonic settings (PM and CIA) derived for sandstones in the Nkapa Formation and emphasizes the reliability of inorganic geochemistry proxies for studying 
tectonic settings linked with sedimentary rocks. The association of the studied shales dominantly with PM and CIA settings indicate terrigenous input. Their $\mathrm{K}_{2} \mathrm{O} / \mathrm{Na}_{2} \mathrm{O}$ ratios varying between $5-28(>1)$ reflect high quartz $\left(\mathrm{SiO}_{2}\right)$ contents.
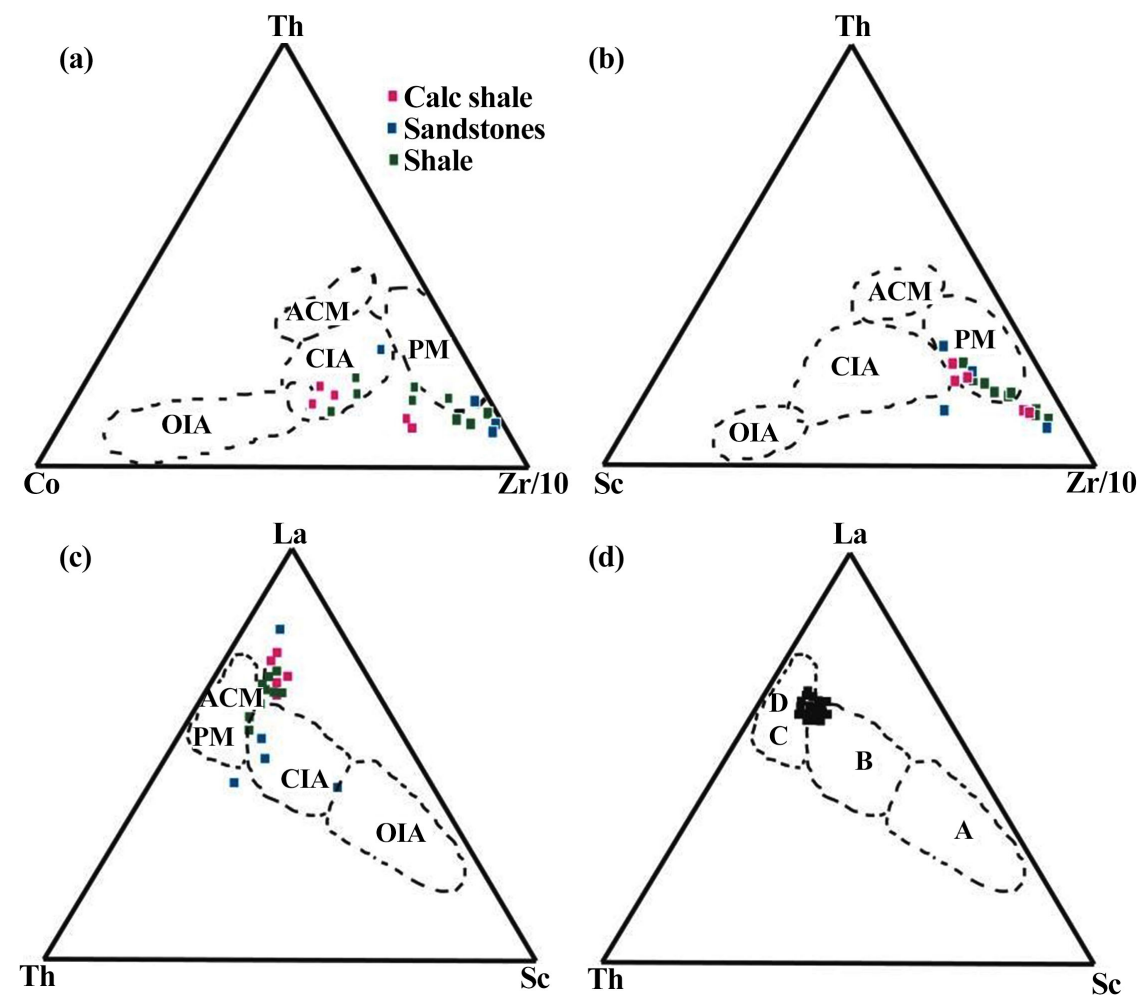

Figure 6. Trace and rare earth elements used to discriminate tectonic settings associated with the Kompina sandstones and shales after [4] based on four settings [4]. The settings include the following: active continental margin (ACM/C), passive margin (PM/D), continental island $\operatorname{arc}(\mathrm{CIA} / \mathrm{B})$ and oceanic island arc (OIA/A). These plots involve the following (a) Th-Sc-Zr/10, (b) Th-Co-Zr/10 and (c) La-Th-Sc and (d) La-Th-Sc showing samples from the Nanxiong Basin after [12].

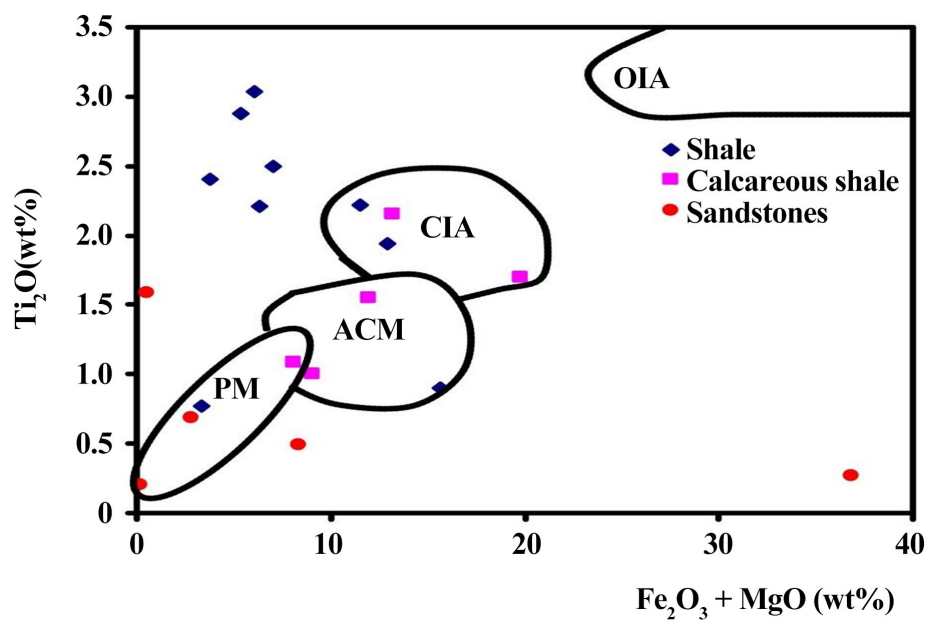

Figure 7. $\mathrm{Fe}_{2} \mathrm{O}_{3}(\mathrm{~T})+\mathrm{MgO}$ vs. $\mathrm{TiO}_{2}$ compositional plot showing the sandstone and shale samples from the Nkapa Formation in different tectonic settings proposed by [3]. 


\section{Palaeoenvironmental Analysis}

\section{Redox Conditions}

Redox-sensitive trace element ratios including $\mathrm{Ni} / \mathrm{Co}$ and $\mathrm{V} /(\mathrm{V}+\mathrm{Ni})$ are usually considered as powerful geochemical indicators commonly employed for environmental discrimination during the deposition of sediments. According to [50], $\mathrm{Ni} / \mathrm{Co}$ ratios of $<5,5-7$ and $>7$ imply oxic, dysoxic and suboxic to anoxic conditions, respectively, during deposition. Relatedly, [51] proposed that for organic matter to accumulate in sediments under euxinic conditions, the $\mathrm{V} /(\mathrm{V}+\mathrm{Ni})$ ratio of the sediments must be $>0.5$.

Figure 8 shows the Ni/Co versus $\mathrm{V} /(\mathrm{V}+\mathrm{Ni})$ data for the samples from the Nkapa Formation in the Douala Basin. The $\mathrm{V} /(\mathrm{V}+\mathrm{Ni})$ ratios from the bulk sediment vary between $(0.53-0.95$ and between $0.66-0.97$ correspondingly for the carbonates and shales. The Ni/Co ratios of the carbonates samples are between 0 - 4.8, with few ranging between 5 - 8.5, whereas ratios for the shale samples are between $1-2.5$, excluding one sample displaying a value of 10.3.

The depositional environment obtained from this study predicts that the sediments are deposited more under an oxic condition. These oxic environments are prone to produce organic matter rich in Type II and III kerogen [21] [52] that can produce more of gas since they are mainly made up of plant materials from the land, good in producing gaseous petroleum when the organic matter becomes mature. However the few samples that ploted in the euxinic environment representing sediments deposited under reducing condition are likely to produce organic matter (planktons, algae) that will release more of oil than gas [53] [54] [55] [56].

The proportionality of $\mathrm{V}$ to $\mathrm{Ni}$, mainly expressed as $\mathrm{V} /(\mathrm{V}+\mathrm{Ni})$, has been utilised to highlight the $\mathrm{Eh}$ and $\mathrm{pH}$ conditions as well as the sulphide concentration in different environments during the deposition of sediments [52].

The solubility of vanadium in natural waters, its extraction from sea water and adsorption onto sediments are influenced by redox conditions [57]. Dissolved vanadium is readily bound to high organometallic complexes [57] or adsorbed onto biogenic materials [58] [59]. During early diagenesis of sediments, vanadium is released from biogenic materials under oxic conditions, whereas this mobilisation is restricted under anoxic conditions [60] [61]. In addition, $\mathrm{Ni}, \mathrm{Cu}$ and $\mathrm{Cr}$ are commonly trapped within organic matter in organic matter-rich sediments [9] [59] [61]. Nevertheless, vanadium accumulation is favoured relative to $\mathrm{Ni}$ in reducing.

The high $\mathrm{V} /(\mathrm{V}+\mathrm{Ni})$ ratios exhibited by the carbonates $(0.53-0.93)$ and shales $(0.53-0.97)$ from the study area (Figure 8$)$ indicate their deposition in dysoxic and anoxic environments. The carbonate and shale samples with $\mathrm{V} /(\mathrm{V}+$ $\mathrm{Ni}$ ) ratios greater than 0.8 indicate the presence of significant $\mathrm{H}_{2} \mathrm{~S}$ in the environment during deposition [48]. Thus, the $\mathrm{V} /(\mathrm{V}+\mathrm{Ni})$ ratios obtained from the shales and carbonates indicate variations in redox conditions during deposition of the sediments in the Nkapa Formation [9]. 


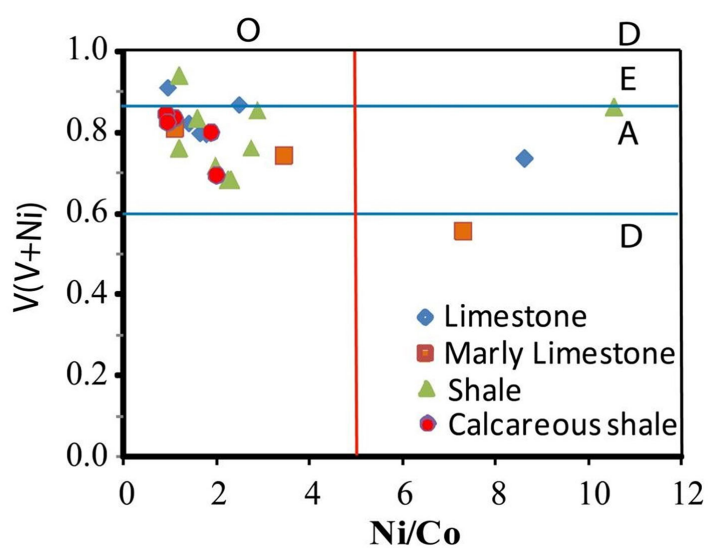

Figure 8. Plot of redox-sensitive trace element ratios: $\mathrm{V} /(\mathrm{V}+\mathrm{Ni})$ vs. $\mathrm{Ni} / \mathrm{Co}$ for the Kompina carbonates and shales (after [48]); where $\mathrm{O}$ = oxic, $\mathrm{D}=$ dysoxic, $\mathrm{A}=$ anoxic, and $\mathrm{E}=$ euxinic environment.

\section{Conclusions}

Sediments from the Nkapa Formation in the north western part of the Douala Basin were evaluated using major, trace and rare earth element geochemistry in order to determine the tectonic setting, provenance, depositional environments and the economic importance of the Tertiary deposited sediments.

From trace and the Rare element plot the sediments from the Kompina area using $\mathrm{Eu} / \mathrm{Eu}^{*}, \mathrm{La} / \mathrm{Sc}, \mathrm{La} / \mathrm{Co}, \mathrm{Th} / \mathrm{Sc}$ and $\mathrm{Th} / \mathrm{Co}$, plotted mainly within the $\mathrm{PM}$ and CIA tectonic settings. While plots using oxides of $\mathrm{Fe}_{2} \mathrm{O}_{3}(\mathrm{~T})+\mathrm{MgO}$ vs. $\mathrm{TiO}_{2}$ were associated with PM, OIA, and CIA provenances. Using F1, F2, Plots reveal that these sediments plotted within the quartzose sedimentary provenance.

The Nkapa Formation sediments were derived from felsic and mafic rocks as shown by Table 4 and Figure 6 . The felsic rocks could have originated from the adjacent migmatitic rocks of the neighbouring basement complex while the mafic rocks might have resulted from mafic volcanic rocks of the Cameroon Volcanic Line in West-Central Africa.

A plot of $\mathrm{V} /(\mathrm{V}+\mathrm{Ni})$ vs. $\mathrm{Ni} / \mathrm{Co}$ depicts that carbonates and shales from the Nkapa Formation were deposited under environmental conditions ranging from oxic through suboxic to anoxic. These environments can be suitable in producing type II and III Kerogene which are prone to produce more gaseous hydrocarbons with minor oil.

Limestones from the studied area at Kompina are rich in Calcium Carbonate above $98.5 \%$. This makes them suitable for use in the chemical industry (e.g., pharmaceutical industries), poultry farming (feed production) and as soil amendments in agriculture to improve plant growth due to their ability to reduce the acid content of soils.

\section{Acknowledgements}

The authors are grateful to Edith Etakah Bate and Bryan Ngatcha for assisting with the quality of the figures. 


\section{Conflicts of Interest}

The authors declare no conflicts of interest regarding the publication of this paper.

\section{References}

[1] Basu, A. (1985) Influence of Climate and Relief on Compositions of Sand Released at Source Area. In: Zuffa, G.G., Ed., Provenance of Arenites, Springer, Berlin, 1-18. https://doi.org/10.1007/978-94-017-2809-6_1

[2] Folk, R.L. (1968) Petrology of Sedimentary Rocks. Hemphill Publishing Co., Austin.

[3] Bhattia, M.R. (1981) Petrology, Geochemistry and Tectonic Setting of Some Flysch Deposits. PHD Thesis, Australia National University, Canberra, 312. (Unpublished)

[4] Bhattia, R.M. and Crook, K.A.W. (1986) Trace Element Characteristics of Greywackes and Tectonic Discrimination of Sedimentary Basins. Contributions to Mineralogy and Petrology, 92, 181-193. https://doi.org/10.1007/BF00375292

[5] Rollinson, H.S. (1993) Using Geochemical Data: Evaluating, Presentation, Interpretation. Chemistry Series. Pearson Prentice Hall, Hoboken, 384 p.

[6] Lee II, Y., Kim, C.J. and Hisada, H. (2007) Depositional and Compositional Controls on Sandstone Diagenesis, the Tetori Group (Middle Jurassic-Early Cretaceous), Central Japan. Sedimentary Geology, 195, 183-202. https://doi.org/10.1016/j.sedgeo.2006.08.011

[7] Roser, B.P. and Korsch, R.J. (1986) Determination of Tectonic Setting of Sandstone-Mud-Stone Suite Using $\mathrm{SiO}_{2}$ Content and $\mathrm{K}_{2} \mathrm{O} / \mathrm{Na}_{2} \mathrm{O}$ Ratio. Journal of Geology, 94, 635-650. https://doi.org/10.1086/629071

[8] Nader, H.F., Haider, T.I., Abdel-Fattah, M. and Abdel-Rahman (2006) Petrographic and Chemical Traits of Cenomanian Platform Carbonates (Central Lebanon): Implications for Depositional Environments. Cretaceous Research, 27, 689-709. https://doi.org/10.1016/j.cretres.2006.03.009

[9] Madhavaraju, J. and Lee II (2009) Geochemistry of the Dalmiapuram Formation of the Uttatur Group (Early Cretaceous), Cauvery Basin, Southeastern India: Implications on Provenance and Paleo-Redox Conditions. Revista Mexicana de Ciencias, 26, 380-394.

[10] Bhattia, M.R. (1983) Plate Tectonic and Geochemical Composition of Sandstones. Journal of Geology, 91, 611-627. https://doi.org/10.1086/628815

[11] Yan, Y., et al. (2007) Geochemistry of the Sedimentary Rocks from the Naxiong Basin, South China and Implications for Provenance, Paleoenvironment and Paleoclimate at the K/T Boundary. Sedimentary Geology, 197, 127-140. https://doi.org/10.1016/j.sedgeo.2006.09.004

[12] Taylor, S.R. and McLennan, S.M. (1985) The Continental Crust: Its Composition and Evolution. Blackwell, Oxford, 321.

[13] Ikhane, P.R., Akintola, A.I., Bankole, S.I. and Oyinboade, Y.T. (2014) Provenance Studies of Sandstone Facies Exposed near Igbile Southwestern Nigeria: Petrographic and Geochemical Approach. Journal of Geography and Geology, 6, 47-68. https://doi.org/10.5539/jgg.v6n2p47

[14] Cameron, N.R. and Ken, W. (1999) Exploration Opportunities in Offshore Deep Water Africa. IBC "Oil and Gas Developments in West Africa", London, 25-26 October 1999, 367-385.

[15] Albrecht, P., Vandenbroucke, M. and Mangengué, M. (1976) Geochemical Studies 
on the Organic Matter from the Douala Basin (Cameroon) I. Evolution of the Extractable Organic Matter and the Formation of Petroleum. Geochimica et Cosmochimica Acta, 40, 791-799. https://doi.org/10.1016/0016-7037(76)90031-4

[16] Brownfield, M.E. and Charpentier, R.R. (2006) Geology and Total Petroleum Systems of the West-Central Coastal Province (7203), West Africa. U.S. Geological Survey Bulletin, 2207-B, 1-52.

[17] Manga, C.S. (2008) Stratigraphy, Structure and Prospectivity of the Southern Onshore Douala Basin, Cameroon-Central Africa. Africa Geoscience Review, 1, 13-37.

[18] Tiziana, L.A., Schmid, E. and Piperi, T. (2009) Recent Discoveries Offshore Douala Basin. Search and Discovery Articles 10, 85.

[19] Ntamark-Nida, M.J., Bourquin, S., Makong, J.C., baudin, F., Mpesse, J.E., Kom-guem, P.B. and Ablolo, G.M. (2010) Sedimentology and Sequence Stratigraphy from Outcrops of Kribi-Campo Sub-Basin: Mundeck Formation (Lower Cretaceous, Southern Cameroon). Journal of African Earth Sciences, 58, 1-18.

https://doi.org/10.1016/j.jafrearsci.2010.01.004

[20] Esue, M.F., Agyingi, C.M., Josepha, F.T. and Anatol, E.D.L. (2021) Geochemistry of Carbonate Clastic Strata in NW Douala Sub-Basin, Cameroon: Implication for Provenance, Tectonics and Source Area Weathering. Journal of Sedimentary Environments, 6, 57-71. https://doi.org/10.1007/s43217-020-00044-1

[21] Ngueutchoua, G., Armel, Z.E., Zandjio, D.D., Djaro, H.B. and Nfada, L.T. (2019) Geochemistry of Cretaceous Fine-Grained Siliciclastic Rocks from Upper Mundeck and Logbajeck Formation, Douala Sub-Basin, South-West Cameroon: Implication of Wethering Intensity. Provenance, Paleoclimate, Redox Condition and Tectonic Settings. Journal of African Earth Sciences, 152, 215-236.

https://doi.org/10.1016/j.jafrearsci.2019.02.021

[22] Ngueutchoua, G., Ngantchu, L.D., Youbi, Ngos, S. and Kabeyene, V.K. (2017) Geochemistry of Cretaceous Mudrocks and Sandstone from Douala-Sub Basin, Kumba Area, South West Cameroon: Constrains on Provenance, Source Rock Weathering, Tectonic Environment. International Journal of Geosciences, 8, 393-424. https://doi.org/10.4236/ijg.2017.84021

[23] Ngaha, P.R.N. (2005) Palynostratigraphy and Reconstruction of the Cretaceous Paleoenvironments of the East of Douala Sedimentary Basin (Cameroon). PhD Thesis, University of Yaounde I, Yaounde, $259 \mathrm{p}$.

[24] Morin, S. (1980) Relief and Hydrography. In: Groupe, J.A., Ed., Atlas of the United Republic of Cameroon. Paris, $72 \mathrm{p}$.

[25] Dumort, J.C. (1968) Rapport spéciale sur les calcaires du Nord-Cameroon. Arch R.G.M. 3.

[26] Meyers, J.B., Rosendahl, B.R., Harrison, G.A.C. and Ding, Z.D. (1998) Deep-Imaging Seismic and Gravity Results from the Offshore Cameroon Volcanic Line, and Speculation of African Hotlines. Tectonophysics, 284, 31-63. https://doi.org/10.1016/S0040-1951(97)00173-X

[27] Meyers, J.B., Rosendahl, B.R. and Groschel-Becker, H. (1996) Deep Penetrating MCS Imaging of the Rift-to-Drift Transition, Offshore Douala and North Gabon Basins, West Africa. Marine and Petroleum Geology, 13, 791-835. https://doi.org/10.1016/0264-8172(96)00030-X

[28] Burke, K. and Whiteman, A.J. (1972) Uplift, Rifting and the Break-Up of Africa: Studies in Earth and Space Science. Geological Society of America, Boulder, Memoir 132, 738-755.

[29] Benkhelil, J., Giresse, P., Paumort, C. and Ngueuchoua, G. (2002) Lithostratigraphic, 
Geophysical and Morpho-Tectonic Studies of the South Cameroon Shelf. Marine and Petroleum Geology, 19, 499-517. https://doi.org/10.1016/S0264-8172(02)00002-8

[30] Klett, T.R., Ahlbrandt, T.A., Schmoker, J.W. and Dolton, G.L. (1997) Ranking of the World's Oil and Gas Provinces by Known Petroleum Volumes. U.S. Geological Survey Open File Report 97-463, One CD-ROM. https://doi.org/10.3133/ofr97463

[31] Clifford, A.C. (1986) African Oil-Past, Present and Future. In: Halbouty, M.T., Ed., Future Petroleum Provinces of the World, American Association of Petroleum Geologists, Tulsa, Memoir 40, 339-372. https://doi.org/10.1306/M40454C13

[32] Ngueuchoua, G. and Giresse, P. (2010) Sand Bodies and Incised Valleys within the Late Quaternary Sanaga-Nyong Delta Complex on the Middle Continental Shelf of Cameroon. Marine and Petroleum Geology, 27, 2173-2188.

https://doi.org/10.1016/j.marpetgeo.2010.06.011

[33] Aloïsi, J.C., Benkhelil, J., Giresse, P. and Ngueuchoua, G. (1995) Etude sismique haute-resolution du précontinent sud-Camerounais: Analyses faciologique et structurale. Comptes Rendus de I Académie des Sciences, 321, 145-152.

[34] Blatt, H., William, B.N., William, B.N. and Brande, S. (1991) Principles of Stratigraphic Analysis. Blackwell Science Publications, Amsterdam, 319-402.

[35] Cohen, H.A. and McClay, K. (1996) Sedimentation and Shale Tectonism of the South-Western Niger Delta Front. Marine and Petroleum Geology, 13, 3. https://doi.org/10.1016/0264-8172(95)00067-4

[36] Doust, H. and Omatsola, E. (1990) Niger Delta. American Association of Petroleum Geologists, Tulsa, Memoir 48, 201-238. https://doi.org/10.1306/M48508C4

[37] Morgan, R. (1999) Ultra-Deep Water Prospectivity in the Niger Delta. IBC Nigeria Energy Summit, London, 15-16 June 1999, 7-8.

[38] Tamfu, S. and Batupe, M. (1995) Geologic Setting, Stratigraphy and Hydrocarbon Habitat of the Douala Basin, Cameroon. NHC News No. 003, Jan-Mar. 95.

[39] Cael, R.M. and Kim, J.Y. (1979) Cameroon P.H.33. Seismic Evolution. Gulf Research and Development Company, Exploration Interpretation Department, Houston, $20 \mathrm{p}$.

[40] SNH (Societé National des hydrocarbure) (1999) Republic of Cameroon, Third License-Licencing Round: Douala-Kribi-Campo Basin. SNH Unpublished Non-Exclusive Report, 4-8.

[41] Abbot, B.M., Walker, J.W. and Prissorsky, J.A. (1978) An Evaluation of the Douala Permit, Cameroon, as an Aid to the 1978 Relinquishment. HTSC Report No. 442A1822, 250.

[42] Nodesa, C. (1971) Lexicon of West Africa Stratigraphic Names (from Togo to An-gola). HTCS Report, 443N0010.

[43] Harrison, D.J. (1993) Limestone: Industrial Minerals Laboratory Manual. British Geological Survey. Technical Report WG/92/29.45.

[44] Gromet, L.P., Dymek, R.F., Haskin, L.A. and Kototev, R.L. (1984) The "North American Shale Composite": Its Compilation, Major and Trace Element Characteristics. Geochimica et Cosmochimica Acta, 48, 2469-2482. https://doi.org/10.1016/0016-7037(84)90298-9

[45] Culler, R.L. (1994) The Controls on the Major and Trace Element Variation of Shales, Siltstones and Sandstones of Pennsylvanian-Permian Age from Uplift Continental Blocks in Colorado to Platform Sediment in Kansas, USA. Geochimica et Cosmo-chimica Acta, 58, 4955-4972. https://doi.org/10.1016/0016-7037(94)90224-0 
[46] Culler, R.L. (2000) The Geochemistry of Shales, Siltstone and Sandstones of Pennsylvanian-Permian Age, Colorado, USA: Implications for Provenance and Metamorphic Studies. Lithos, 51, 305-327. https://doi.org/10.1016/S0024-4937(99)00063-8

[47] Wronkiewicz, D.J. and Condie, K.C. (1990) Geochemistry and Mineralogy of Sediments from the Venter Drop and Traveaal Supergroup, South Africa: Cratonic Evolution during Early Proterozoic. Geochimica et Cosmochimica Acta, 54, 343-354. https://doi.org/10.1016/0016-7037(90)90323-D

[48] Cox, R., Low, D.R. and Culler, R.L. (1995) The Influence of Sediment Recycling and Basement Composition on Evolution of Mudrock Chemistry in the Southwestern United States. Geochimica et Cosmochimica Acta, 59, 2919-2940. https://doi.org/10.1016/0016-7037(95)00185-9

[49] Cullers, R.L. (1995) The Controls on the Major and Trace Element Evolution of Shales, Siltstones and Sandstones of the Ordovician to Tertiary Age in the Wet Mountain Region, Colorado, U.S.A. Chemical Geology, 123, 107-131. https://doi.org/10.1016/0009-2541(95)00050-V

[50] McLennan, S.M. (1989) Rare Earth Elements in Sedimentary Rocks: Influence of Provenance and Sedimentary Processes. Reviews in Mineralogy, 21, 169-200. https://doi.org/10.1515/9781501509032-010

[51] Jones and Manning, D.A.C. (1994) Comparison of Geochemical Indices Used for the Interpretation of Palaeo-Redox Conditions in Ancient Mudstones. Chemical Geology, 111, 111-129. https://doi.org/10.1016/0009-2541(94)90085-X

[52] Kenfack, P.L., Ngaha, P.R.N., Ekodeck, G.E. and Ngueutchoua, G. (2012) Mineralogic Characterization of Petroleum Potential of Clays (Shales of the Nkapa Formation (Paleocene-Eocene) in the Doula Sedimentary Sub-Basin (South-West Cameroon). International Journal of Geosciences, 3, 696-709.

https://doi.org/10.4236/ijg.2012.34070

[53] Bitchong, M., Adatte, T.T.F.N. and Ngon, G.F. (2021) Palynology, Mineralogy and Geochemistry of Sediments in Toned Locality, Northern Part of the Douala Sub-Basin, Cameroon, Central Africa: Implication of Paleoenvironment. Geoscience Journal, 25, 299-319. https://doi.org/10.1007/s12303-020-0021-z

[54] Mbesse, C., Roche, O.E. and Ngos III, S. (2012) La limite Paleocene-Eocene Dans le basin de Douala (Cameroon). Biostratigraphic et essai de reconstruction de Paleoenvironments par L'etude de Dinoflagelles. GeoEcon-Trop, 36, 83-119.

[55] Hatch, J.R. and Leventhal, J.S. (1992) Relationship between Inferred Redox Potential of the Depositional Environment and Geochemistry of the Upper Pennsylvanian (Missourian) Stark Shale Member of the Dennis Limestone, Wabaunsee County, Kansan, USA. Chemical Geology, 99, 65-82. https://doi.org/10.1016/0009-2541(92)90031-Y

[56] Tribovillard, N.P., Desprairies, A., Lallier-Verges, E., Bertrand, P., Moureau, N., Ramdani, A. and Ramanampisoa, L. (1994) Geochemical Study of Organic-Matter Rich Cycles from the Kimmeridge Clay Formation of Yorkshire (UK): Productivity versus Anoxia. Paleogeography, Paleoclimatology, Paleoecology, 108, 165-181. https://doi.org/10.1016/0031-0182(94)90028-0

[57] Lewan, M.D. and Maynard, J.B. (1982) Factors Controlling Enrichment of Vanadium and Nickel in the Bitumen of Organic Sedimentary Rocks. Geochimica et Cosmochimica, 46, 2547-2560. https://doi.org/10.1016/0016-7037(82)90377-5

[58] Prange, A. and Krembling, K. (1985) Distribution of Dissolved Molybdenum, Uranium and Vanadium in Baltic Sea Waters. Marine Geology, 16, 259-274. 
https://doi.org/10.1016/0304-4203(85)90066-0

[59] Leventhal, J.S. and Hosterman, J.W. (1982) Chemical and Mineralogical Analysis of Devonian Black-Shale Samples from Martin County, Kentucky; Carroll and Washington Counties, Ohio; Wise County, Virginia; and Overton County, Tennessee, U.S.A. Chemical Geology, 37, 239-264.

https://doi.org/10.1016/0009-2541(82)90081-X

[60] Gilkson, M., Chapell, B.W., Freeman, R.S. and Webber, E. (1985) Trace Elements in Oil Shales, Their Source and Organic Association with Particular Reference to Australian Deposits. Chemical Geology, 53, 155-187.

https://doi.org/10.1016/0009-2541(85)90028-2

[61] Gromet, L.P., Haskin, L.A., Korotev, R.L. and Dymek, R.F. (1990) The "North American Shale Composite": Its Compilation, Major and Trace Element Characteristics. Geochimica et Cosmochimica Acta, 48, 2469-2482.

https://doi.org/10.1016/0016-7037(84)90298-9 